\title{
Graphdiyne: from Preparation to Biomedical Applications
}

\author{
LI Xiaodan\#, GUO Mengyu\# and CHEN Chunying ${ }^{\bowtie}$
}

Received August 31, 2021

Accepted September 21, 2021

(C) Jilin University, The Editorial Department of Chemical Research in Chinese Universities and Springer-Verlag $\mathrm{GmbH}$
G raphdiyne(GDY) is a kind of two-dimensional carbon nanomaterial with specific configurations of $s p$ and $s p^{2}$ carbon atoms. The key progress in the preparation and application of GDY is bringing carbon materials to a brand-new level. Here, the various properties and structures of GDY are introduced, including the existing strategies for the preparation and modification of GDY. In particular, GDY has gradually emerged in the field of life sciences with its unique properties and performance, therefore, the development of biomedical applications of GDY is further summarized. Finally, the challenges of GDY toward future biomedical applications are discussed.

Keywords Graphdiyne(GDY); Modification; Biomedical application

\section{Introduction}

Carbon has four electrons in the outer layer of nucleus, so it can form three common hybrid structures $\left(s p, s p^{2}, s p^{3}\right)^{[1,2]}$. Furthermore, abundant carbon allotropes can be formed through covalent bonds(Fig.1). After the diamond(sp $\left.{ }^{3}\right)$ and graphite $\left(s p^{2}\right)$ have been known, fullerene $\left(\mathrm{C}_{60}, s p^{2.28}\right)$, a spherical carbon molecule composed of 12 five-membered rings and 20 six-membered rings, was developed in 1985 by Kroto et al. ${ }^{[3]}$ Driven by fullerene research, in 1991, a more peculiar carbon structure, carbon nanotube, was discovered by Lijima of $\mathrm{NEC}^{[4]}$, which further deepened people's understanding of low dimensional carbon materials. In 2004, Geim et al. ${ }^{[5]}$ firstly prepared a two-dimensional(2D) material, graphene, which was composed of $s p^{2}$ hybrid carbon atoms arranged in a hexagon honeycomb structure periodically and connected with each other in the form of covalent bond. Graphene is a zero-band gap semiconductor material with excellent electrical properties $^{[6]}$. However, the energy band and performance regulation of graphene is still an unsolved difficulty ${ }^{[7,8]}$. Therefore, we are looking for a new type of carbon-based 2D structure with both excellent band gap and higher carrier mobility.

As early as 1987, Baughman et al. ${ }^{[9]}$ proposed a kind of two-dimensional allotrope material of carbon, which is formed by the arrangement of carbon atoms in $s p$ and $s p^{2}$ hybrid states

\footnotetext{
$\triangle$ CHEN Chunying

chenchy@nanoctr.cn

\# These authors contributed equally to this work.

CAS Key Laboratory of Biomedical Effects of Nanomaterials and Nanosafety \& CAS Center for Excellence in Nanoscience, National Center for Nanoscience and Technology of China, Beijing 100190, P. R. China
}

according to a certain periodic rule, which was called graphynes. This new carbon allotrope, especially graphdiyne (GDY), has different active $s p$ hybrid carbon and special conjugated structure from graphene, which has aroused researchers' exploration of its properties. In 1997, GDY was first proposed by Haley et al. ${ }^{[10]}$, then it was predicted to be a semiconductor material with direct band gap $(0.46 \mathrm{eV})$ and high room temperature carrier mobility $\left(10^{4}-10^{5} \mathrm{~cm}^{2} \cdot \mathrm{V}^{-1} \cdot \mathrm{s}^{-1}\right)^{[11]}$. However, due to the instability of its monomers and the existence of carbon-carbon triple bonds in the structure, the experimental synthesis of graphynes presents a huge challenge. It was not until 2010 that GDY was first synthesized by $\mathrm{Li}$ et al. ${ }^{[12]}$ using the in situ Glaser coupling reaction of hexaethynylbenzene(HEB) monomer on the copper substrate. This new type of carbon-based 2D atomic crystal material was synthesized on the copper substrate by solution chemistry. Atomic force microscopy(AFM) results showed that the thickness of the film was about $1 \mu \mathrm{m}$. The conductivity is $2.516 \times 10^{-4} \mathrm{~S} / \mathrm{m}$. The success of the preparation of GDY opened up the research enthusiasm of GDY. The discovery of carbon materials left Chinese footprints for the first time, which laid an important foundation for the follow-up research of new carbon materials.

After that, scientists are committed to the exploration of high-quality preparation of GDY and broadening the applications of its special properties. In biomedical field, taking special pore structure, $\pi-\pi$ conjugated systems, special photoresponse properties and geometrical properties as advantages, GDY has new understanding and applications in

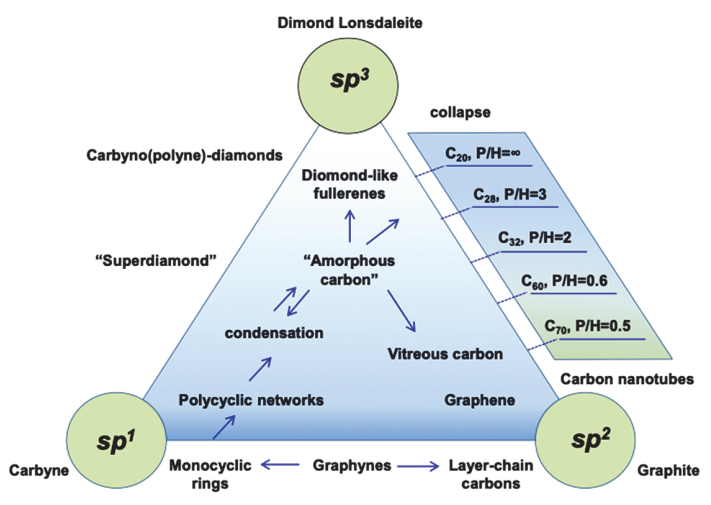

Fig.1 Schematic illustration of the family of carbon allotropes ${ }^{[1]}$ Reprinted with permission from ref.[1]. Copyright 1997, Elsevier Ltd. 
biomedical fields, such as tumor therapy, antibacterial, radiation protection, bionics, etc. ${ }^{[13]}$.

Herein, various properties, structures, and preparation and modification methods of GDY are introduced, including the development of GDY in different aspects of biomedical applications in recent years. Fig. 2 shows the main idea for this review.

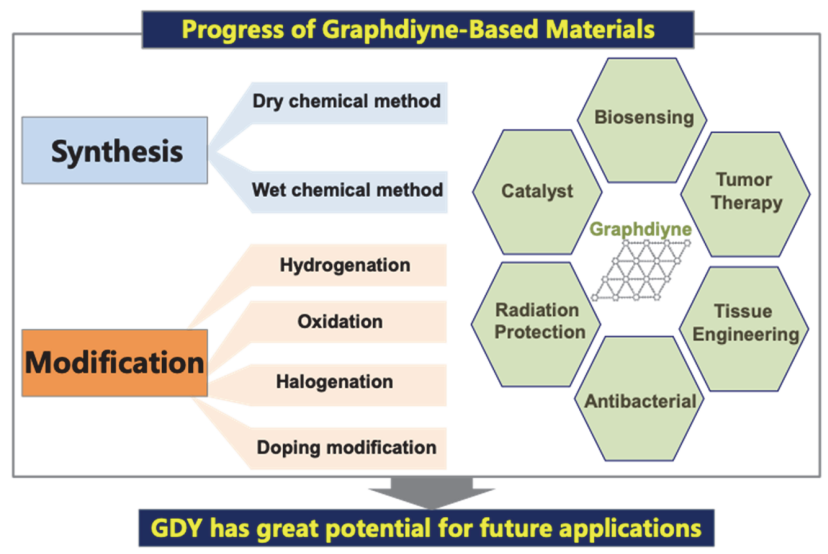

Fig.2 Current progress in preparation, modification and application of GDY

\section{Classification, Structure Characterization and Properties of Graphynes}

\subsection{Classification}

$s p$ and $s p^{2}$ carbon atoms can be connected by covalent bond according to certain rules, which can construct a variety of different 2D planar structures. They form a large family of graphyne, which can be regarded as a kind of 2D periodic structure formed by two $s p^{2}$ carbon atoms connected by $n$ ' $-\mathrm{C} \equiv \mathrm{C}-$ '. We can simply classify these materials according to the number of alkyne bonds ' $n$ ' connecting two $s p^{2}$ carbon atoms, which are called 'Graphyne', 'Graphdiyne' and 'Graphyne- $n$ ', respectively ${ }^{[14]}$.

The nomenclature of graphyne is based on the structural units, and can be divided into systematic nomenclature and customary nomenclature. Systematic nomenclature is a nomenclature for graphyne based on the number of carbon atoms in the ring of graphyne structure ${ }^{[14-17]}$. As shown in Fig.3, graphyne can be named as $\alpha, \beta, \gamma$-Graphyne. Among them, $\alpha$-Graphyne can be regarded as a carbon-carbon triple bond $(-\mathrm{C} \equiv \mathrm{C}-$ ) inserted between each $-\mathrm{C}-\mathrm{C}-$ bond in a graphyne structure, $\beta$-Graphyne is a carbon-carbon triple bond $(-\mathrm{C} \equiv \mathrm{C}-$ ), which is inserted between two-thirds of the $-\mathrm{C}-\mathrm{C}-$ bonds, and $\gamma$-Graphyne is a carbon-carbon triple bond $(-\mathrm{C} \equiv \mathrm{C}-$ ), which is inserted between one third of the $-\mathrm{C}-\mathrm{C}-$ bonds. With the development of biomedical sciences, as the first prepared graphyne, GDY[Fig.3(G)] also has huge application potential in biological field.

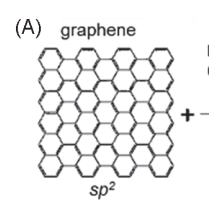

(B)

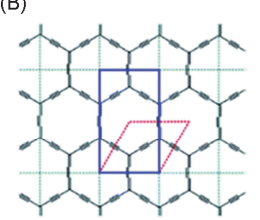

(E)

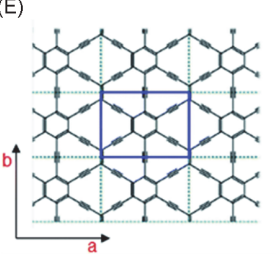

Fig. 3 Structures of graphyne family ${ }^{[14]}$

(A) Schematic structures of graphene and graphynes; (B) $\alpha$-Graphyne; (C) $\beta$ Graphyne; (D) $\gamma$-Graphyne; (E) 6,6,12-Graphyne; (F) $\beta$-Graphdiyne; (G) GDY. The rhombus consists of a red dotted line in $(B-D) ;(F)$ and $(G)$ show the primitive cell, indicating the smallest repeating unit for constituting graphynes. The blue rectangular box in this figure represents a super-packet cell for charge transport calculations. The two perpendicular sides of the rectangle are defined as two directions denoted as $a$ and $b$ directions, respectively.

Reprinted with permission from ref.[14]. Copyright 2019, Royal Society of Chemistry.

\subsection{Structure Characterization and Properties}

Graphynes have well-distributed pore structures and large $\pi$ conjugated systems, endowing them with potential applications in biosensors, biological imaging, cancer treatment, antibacterial and radiation protection ${ }^{[13]}$. In these biomedical applications, graphyne-based materials show better performance and higher stability than other carbon based materials. Huang et al. ${ }^{[18]}$ calculated the Gibbs free energy of various carbon allotropes with graphyne and hydrogen as reactants, and found that the Gibbs free energy of graphynes was $0.803 \mathrm{eV}$, which was larger than those of diamond $(-0.022 \mathrm{eV}), \mathrm{C}_{60}(0.364 \mathrm{eV})$ and carbon nanotube (6, 6) $(0.014 \mathrm{eV})$. These results indicated that graphynes were the most unstable kind of carbon allotropes.

Properties determine applications. Graphynes benefit from the unique structure, such as GDY, which can be applied in many fields. Zhang et al. ${ }^{[19]}$ compared the fracture stress, strain and Young's modulus of several kinds of graphynes and graphene. They found that the introduction of $-\mathrm{C} \equiv \mathrm{C}-$ reduced the rigidity and enhanced the flexibility of graphynes, so they could produce greater deformation under stress. In addition, Shao et al. ${ }^{[20]}$ found that the mechanical properties of graphynes were related to temperature. With the increase of temperature, the mechanical properties of graphynes decreased obviously.

Electrical property is one of the most attractive properties 

could reach $2.1 \times 10^{5} \mathrm{~cm}^{2} \cdot \mathrm{V}^{-1} \cdot \mathrm{s}^{-1}$. Similar to other carbon materials, physical properties of graphynes can be changed through chemical doping, such as the band gap adjusting ${ }^{[21]}$.

Since GDY is a semiconductor with a direct band-gap, the optical performance can be studied by adjusting the inherent microstructure(such as sizes, layer number, stack configuration, etc.), chemical doping, external strain and electric field, etc. GDY possesses high hole mobility and narrow band gap, so it can be a new kind of carbon-based photoluminescence(PL) material, and can have many potential applications, such as UV photodetectors and bioimaging system $^{[22]}$.

Chen et al. ${ }^{[23]}$ and Luo et al. ${ }^{[24]}$ revealed the effect of graphyne allotropes stacking structure on the optical properties by density functional theory, which provided a theoretical basis for the structural identification of graphynebased photocatalyst. According to the results of many studies, the light absorption of GDY was related to its layer number and stacking arrangement. GDY also showed a high fluorescence quenching ability, which could be used as a platform for fluorescence sensing, and the quenching ability of oxidized GDY was higher than that of GDY ${ }^{[25-28]}$.

\section{Synthesis of Graphynes}

It is critical to understand the intrinsic structure and physical properties of GDY for its basic research and practical application. However, due to the difficulty of obtaining high quality GDY up to now, the current theoretical research is far beyond experimental research. So far, in order to further study the intrinsic structure and properties of GDY, researchers have devoted themselves to the controllable synthesis of highquality GDY with single or few layers.

In the past decade, various approaches have been developed to synthesize GDY with controllable layer and different morphologies, such as GDY films ${ }^{[12]}$, nanowalls ${ }^{[14]}$, nanoribbons ${ }^{[11,18]}$, nanosheets ${ }^{[10,11]}$, nanotubes ${ }^{[29]}$ and free standing three-dimensional(3D) GDY[26]. Kong et al.[30] summarized the gaps between reality and ideality of GDY, and revealed that the synthesis of GDY needed a long way to go(Fig.4). Generally, there are two traditional strategies for the preparation of $2 \mathrm{D}$ nanomaterials: one is the bottom-up category starting from existing precursors/monomers, the other is the top-down preparation method starting from block GDY, which has not been realized at present. So, the existing synthesis technology of GDY can be divided into dry chemistry and wet chemistry(Fig.5). Moreover, the

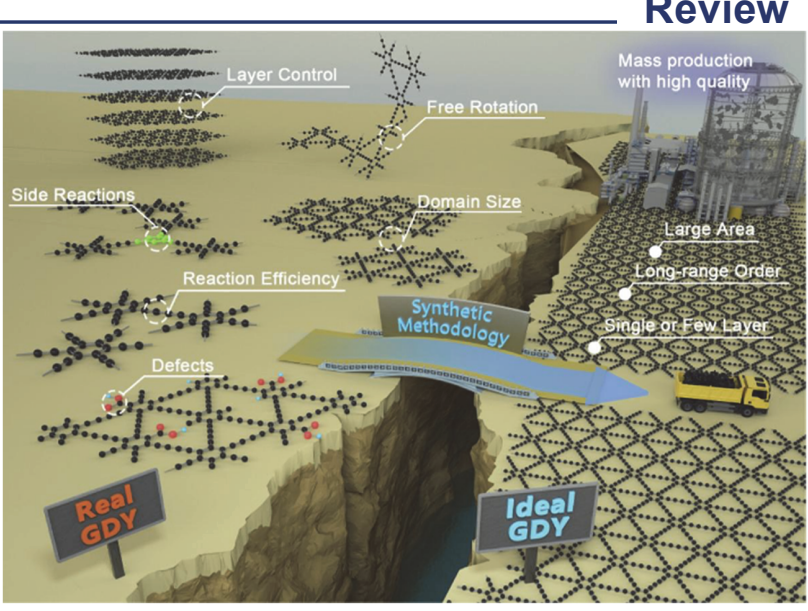

Fig.4 Schematic illustration of the gap between reality and ideality of GDY[30]

Black ball and green ball, carbon atom; red ball, oxygen atom; blue ball, hydrogen atom.

Reprinted with permission from ref.[30]. Copyright 2020, Elsevier Ltd.

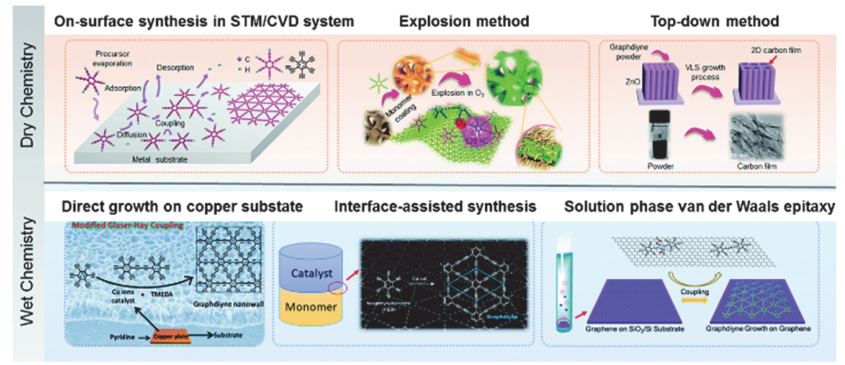

\section{Fig.5 Synthesis of GDY[14]}

Reprinted with permission from ref.[14]. Copyright 2019, Royal Society of Chemistry.

functionalized GDY and other GDY-based materials are synthesized with different novel strategies, especially for its deep use in biomedical area. In order to obtain high-quality GDY with high crystallinity and low defects, the preparation methods have emerged one after another ${ }^{[10,31,32]}$.

Dry chemical synthesis is based on methods of controlled synthesis under strict, non-solution conditions. At present, the dry synthesis methods of GDY include surface preparation under high vacuum of STM, chemical vapor deposition synthesis and explosion method ${ }^{[33-38]}$. The above synthesis method of GDY is precise, but it is not suitable for mass production and commercial application. Due to the problems of monomer stability, rotation of carbon-carbon single bond and low specific surface area of substrate, the quality control and preparation efficiency of GDY are limited. Synthesizing GDY is via the wet chemical routes possesses: Cu-surfacemediated synthesis, interface-assisted synthesis, and solutionphase van der Waals epitaxy. According to different functional groups, the methods for preparing GDY based on coupling reaction can be roughly divided into two categories: the first is the homogeneous coupling reaction between monomers and terminal alkynyl groups. Among them, the most commonly used are Glaser, Glaser Hay and Eglinton coupling 

fraction $)^{[46]}$, it is still a great challenge to synthesize single/ few whole process and usually require additional heating. The other type is homocoupling of terminal alkynyl-silane groups, which can enhance the reaction efficiency without deprotection(such as Hiyama coupling) ${ }^{[41]}$. Due to the low efficiency and various by-products, it is still aimed at developing new precursors with high stability and high reactivity to improve the preparation of GDY ${ }^{[42]}$. The coupling reaction mechanism is shown in Fig.6.

It is also necessary to develop a method for directly growing GDY with specific structure and properties to a specific target substrate. This method can keep the intrinsic properties and aggregation morphology of GDY unchanged, and GDY combined with different functional substrates can realize the construction of a variety of functional materials, greatly expanding the application field of GDY[38,43].

Confined synthesis strategy uses strategies, such as concentration diffusion, template effect, interface restriction or temperature gradient to limit the target substrate or react in a certain space, which can prepare 2D materials. These confined strategies can deal with the poor stability of the monomer, the lower reaction efficiency and side reactions, and also the single bond between the diacetylenefree rotation ${ }^{[37,44,45]}$.
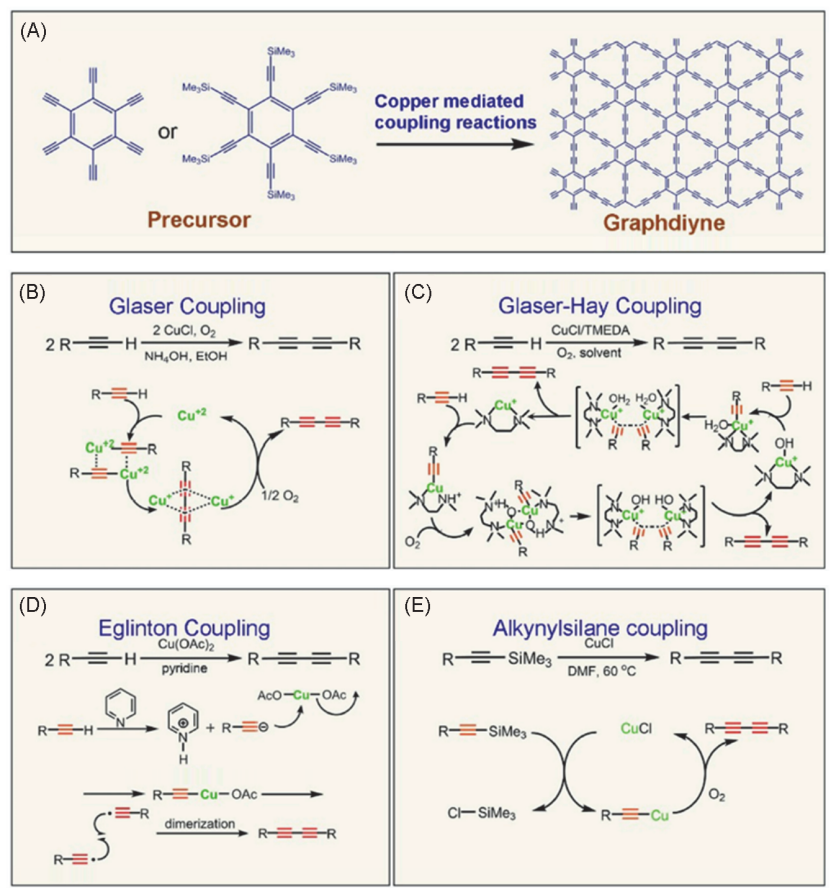

Fig.6 Synthetic route and Cu-mediated acetylenic coupling reactions toward GDY ${ }^{[30]}$

(A) Concluded synthetic route for GDY based on HEB or HEB-TMS precursor; (B) proposed mechanism for classical Glaser coupling reaction; (C) proposed mechanism for Glaser-Hay coupling reaction; (D) proposed mechanism for Eglinton coupling reaction; $(E)$ proposed mechanism for alkynyl-silane coupling reaction.

Reprinted with permission from ref.[30]. Copyright 2020, Elsevier Ltd.

Although bulk GDY can be exfoliated into single- or layer GDY single crystal films that can keep their twodimensional material characteristics. The main problems are as follows.

1) The single bond connecting benzene ring and alkyne bond in the monomer can rotate freely, which will lead to the formation of highly branched or cross connected threedimensional skeleton structure in the reaction process, so we cannot get the expected high-quality layered crystal samples.

2) The traditional epitaxial substrate surface has dangling bonds, which makes the epitaxial layer and substrate have strong interaction, so the lattice of epitaxial layer and substrate should be matched.

3) Due to the existence of ES barrier, the 'step-down' diffusion of monomers adsorbed on the epitaxial layer is inhibited, which will lead to the aggregation and nucleation of monomers on the surface of epitaxial layer, resulting in the formation of thick layer GDY samples(layer by layer stacking out of plane growth mode).

\section{Strategies for Graphdiyne Modification}

The poor solubility and processing properties of GDY are the main obstacles that limit its applications, especially in biological systems. The high activity of the acetylene bond unit in GDY provides a good platform for its chemical modification and doping. At present, progress has been made in nonmetallic heteroatom doping, metal atom modification, and surface modification. The photoelectric properties, energy storage, conversion efficiency, and catalytic performance of GDY are improved a lot. In particular, many important results have been achieved in theoretical research, and experimental work has gradually increased in recent years, but the research on GDY is still in the preliminary stage of development.

Herein, we made a more comprehensive summary and discussion of the current modification strategies of GDY, which will also provide gist for the wider application of graphyne allotropes in the future.

\subsection{Chemical Surface Modification}

At present, the main bottleneck hindering researchers' further study is the poor solubility and processability of GDY. But there is still a long way to explore the preparation of higher quality GDY, and researchers prefer to use the alkyne bond/sub nanopore and the semiconductor energy band structure of GDY for chemical functionalization, and the fact also proves the importance of chemical modification. 


\subsubsection{Hydrogenation}

Hydrogen modified GDY is often called hydrogenated GDY. The $s p$ and $s p^{2}$ hybridization and large $\pi-\pi$ conjugated system of GDY and its derivatives can provide more adsorption space for $\mathrm{H}_{2}$ adsorption. GDY can covalently bind with one or more hydrogen atoms to transform $s p^{2}$ and $s p$ carbon atoms into $s p^{3}$ or $s p^{2}$ carbon atoms, thus laying a foundation for the preparation of new carbon allotropes. At present, the study of $\gamma$-GDY-H system is mainly focused on two aspects: (I) the effect of hydrogen adsorption on the regulation of the properties of $\gamma$-GDY itself, and (II) the use of $\gamma$-GDY for hydrogen storage.

Regarding the influence of hydrogenation on the intrinsic properties of GDY, the researchers found that the increase in the degree of hydrogenation has a negative impact on the mechanical properties of GDY from the perspective of mechanical properties ${ }^{[47]}$. Some researchers also simulated the combination of hydrogen atom and GDY with orbital acetylene bond. In the research, it was found that the priority of hydrogen atom binding to $s p$ and $s p^{2}$ acetylenic bonds was different under different circumstances ${ }^{[48,49]}$.

$\mathrm{Li}$ et al. ${ }^{[50]}$ used first-principles systems to study the dynamic stability and electronic structure changes of hydrogenated GDY. By analyzing the enthalpy of formation, they pointed out that the hydrogenation configuration $(\mathrm{eHH})$ containing only $s p^{3}$ hybrid carbon atoms was more stable than the hydrogenation configuration $(\mathrm{eH})$ of each carbon atom passivated by a single hydrogen atom. However, the eHH of each carbon atom passivated by a single hydrogen atom is more stable. The temperature dependence of the Mholtz free energy indicates that $\mathrm{eH}$ is more favorable than $\mathrm{eHH}$ below $670 \mathrm{~K}$. The phonon spectrum calculation based on DFT confirmed the dynamic stability of $\mathrm{eHH}$ and $\mathrm{eH}$. More particularly interesting is that as the hydrogenation concentration increases, the band gap of $\gamma$-Graphyne undergoes a transition from direct band gap to indirect band gap and to direct band gap again. The results showed that eHH was beneficial to the application of deep ultraviolet lightemitting devices. As the hydrogenation concentration of $\gamma$ GDY increases, its band gap undergoes a direct-indirect-direct transition. This feature similar to hexagonal boron nitride (h$\mathrm{BN}$ ) is very conducive to its application in the field of deep ultraviolet light emitting devices.

\subsubsection{Oxidation}

Oxygen modification is a promising method for functionalizing GDY to achieve specified properties. Most studies have focused on the mechanism of oxidation on the regulation of band structure and photoelectric properties of
GDY. In the biological field, the oxidation treatment of GDY is often used to improve the hydrophobicity and dispersion.

Zhang et al. ${ }^{[51]}$ further studied the effect of the coverage of oxygen-containing functional groups on the structure and properties of GDY band, which provided a basis for the oxidative modification of GDY. Mohajeri et al. ${ }^{[52,53]}$ found that the band gap of $\gamma$-GDY modified by oxygen-containing groups(carbonyl, hydroxyl, carboxyl, epoxy, etc.) would change with the type and concentration of oxygen-containing groups( $0.53-1.51 \mathrm{eV})$. Different oxygen-containing functional groups migrate the Fermi level of the energy band structure of GDY through electron hole doping effect, and then adjust the band gap of GDYO to meet the conditions of photocatalysis. The specific calculation showed that the functionalization of GDY sheet containing $\mathrm{C}=\mathrm{O}$ group made HOMO move towards high energy, but LUMO change little. In contrast, the - $\mathrm{COOH}$ - group was more effective for LUMO and transferred LUMO to a lower energy. These shifts reduced the threshold of optical transition between HOMO region and LUMO region, and the peak appeared in a low energy region. Above are the research results on the modification of GDY by oxidation found in the current research.

In recent years, many researchers are also committed to exploring the application of GDY in the field of biology ${ }^{[13]}$. $\mathrm{Li}$ et al. ${ }^{[54-56]}$ applied GDY and GDYO to many fields including the biological research. In order to increase the compatibility and dispersion of GDY in the biological aqueous environment, the researchers obtained GDYO through oxidation treatment to ensure better effect. More recently, Yan et al. ${ }^{[57]}$ made further study of GDYO, and they gave new comments for the unique 2D GDYO. Wang et al. ${ }^{[58]}$ used the doping of oxygen-containing functional groups to tune the valence band maximum(VBM) of GDYO from $5.46 \mathrm{eV}$ of GDY to $5.70 \mathrm{eV}$. This VBM position made GDYO nanosheets have sufficient thermodynamic driving force for photocatalytic $\mathrm{O}_{2}$ generation, so GDYO could alleviate diffusion and perfusion limited by hypoxia at the same time, and showed effective PDT tumor ablation.

Currently, GDY applied in the field of biology has been commonly modified by oxidation to increase the dispersion and hydrophilicity of its basic aqueous solution system. Other studies have also shown that GDYO has more excellent biocompatibility and safety, and lower biotoxicity compared to graphene oxide(GO) and other carbon materials ${ }^{[57,59]}$.

\subsubsection{Halogenation}

Although GDY has many different allotropes, they are all composed of $s p$ and $s p^{2}$ hybrid carbon atoms. In $\gamma$-GDY, for example, the $s p$ hybrid carbon atoms form an alkyne bond, which is very beneficial to the addition reaction with halogen. Unlike graphene, which can only be fluorinated, GDY can be 
fluorinated and halogenated with chlorine, bromine and iodine. At the same time, the reaction products are all the $s p^{2}$ hybrid carbon atoms replaced by halogen, not the fluorine $s p^{3}$ hybrid carbon atoms obtained by graphene.

The halogenation reaction of $\gamma$-GDY was studied by Lee's group ${ }^{[60]}$. The study showed that the halogen atom(M) was preferentially combined with the $s p$ hybrid carbon atom when the halogen atom was added to the GDY, with one halogen atom above the ring plane and the other halogen atom below the ring plane. According to the theoretical calculation, the halogen atoms in the halogenation reaction can only be outside the original $\gamma$-GDY plane, but not inside the plane, which is mainly because of the larger atomic radius of the halogen atoms, resulting in a larger steric resistance.

Compared with the chlorination, bromination and iodization of GDY, the fluorination reaction of GDY is very different. It is found that $\gamma$-GDY can fully open the triple bond and get the form of $s p^{3}$ hybridization when the fluorination reaction occurs ${ }^{[21]}$. Li et al. ${ }^{[61]}$ prepared two-dimensional material F-GDY by solution method through $\mathrm{C}-\mathrm{C}$ coupling reaction. F-GDY flexible electrode had excellent electrochemical performance due to its high conductivity, high specific surface area, short lithium-ion diffusion distance and a large number of active lithium storage sites. The $\mathrm{C}-\mathrm{F}$ bond of F-GDY gives the F-GDY membrane excellent stability and compatibility with the electrolyte.

\subsection{Doping Modification}

At present, the prepared GDY still has defects, which limits its further development. However, the exploration of the preparation process is slow, and the functional modification (like doping) of GDY on the existing basis is one of the best ways to improving the application limitations of GDY.

\subsubsection{Vacancy Defect Doping}

The effect of defects(such as vacancy defects) on materials may cause various changes in properties. Various studies on preventing vacancy defects can change the electronic properties of carbon nanomaterials, such as the electronic properties of GDY and spin technology.

Kim et al. ${ }^{[62]}$ revealed using density function theory calculation, compared with pure GDY sheet, modified graphite sheet acetylene vacancy defects showed stronger sulfur dioxide $\left(\mathrm{SO}_{2}\right)$ adsorption, and the active position on adsorption of $\mathrm{SO}_{2}$ caused deformation structure of GDY sheet of acetylene and electronic configuration, which can lead to the change of electrical conductivity and magnetic GDY sheet of acetylene. These characteristics indicated that the vacancy defect modified graphite monoacetylene had a wide range of potential applications in electronic devices, magnetic materials and gas sensors.

\subsubsection{Doping of Non-metallic Elements}

The doping of heteroatoms makes it an effective method to adjust and improve the electronic structure and surface chemical activity of GDY itself through the electronegativity difference between carbon and heteroatoms. It is also considered to be a quick and effective way to preparing new graphyne derivatives. The most common heteroatoms used for doping modified graphyne allotropes include N, B, P, F, S, etc. ${ }^{[50]}$.

Single atom doping: doping of a single element can not only effectively improve the catalytic performance of oxygen reduction of GDY, but also has good storage functions of electric energy and hydrogen energy.

Prof. Chen et al. ${ }^{[63]}$ reported B, N single-doped GDY and its catalytic effect on oxygen reduction reaction. The results showed that when pyridine was used as the probe, the Bsubstituted GDY was a good substrate for the Raman enhanced spectra, and was beneficial to the catalytic oxygen reduction reaction. The doping of $\mathrm{B}$ atom will introduce the hole, resulting in the local center of positive charge, and change the electronic structure of the GDY plane. The $\mathrm{N}$ atom in pyridine, which is negatively charged, will be attracted to these positive centers by electrostatic attraction. The strong bond between pyridine and GDY affects the mechanism of some reactions. For example, electron transport between the two contributes to Raman enhancement under incident light.

In addition to the common doping of nitrogen, boron, chlorine and other elements mentioned above, the introduction of oxygen-containing functional groups into GDY can also be considered as oxygen doping from another perspective, which also has a great regulatory influence on the adsorption capacity and energy storage properties of GDY[63-69].

Multi-element doping: single atom doping can give different properties of GDY, and multiple heteroatom codoping can complement the limitation of single atom doping performance regulation, and more effectively regulate the electrical properties and optical properties of GDY[66].

Kang's group ${ }^{[70]}$ showed that the co-doping of $\mathrm{B}, \mathrm{N}$ and $\mathrm{O}$ elements could effectively change the electrical properties of GDY, and the doping position of the three elements played a crucial role in the process of regulating its electrical properties. The results showed that the electronic structure of $\alpha$-GDY was mainly affected by the doping position of $\mathrm{B} / \mathrm{O}$. The electronic structure of $\beta$-GDY is significantly dependent on the doping position of $\mathrm{O}$ doping. The electronic structure of $\gamma$-GDY depends mainly on the location of $\mathrm{B}$ and $\mathrm{O}$ doping. 

positively charged $\mathrm{B}$ atom contributed and transferred its energy state to a higher energy level in the LUMO state, causing the band gap to widen and changing the optical response.

At present, there are various studies on multi-atom doping of GDY, and the most frequently studied is the codoping of nitrogen, boron, oxygen and other elements. On this basis, adding some metal atoms, such as manganese also has very interesting research findings.

Chemical synthesis doping: chemical synthesis doping can be directly doped and modified once in the process of preparing GDY, which not only makes the doping more comprehensive, but also avoids the damage to the material itself during the secondary modification. After Li's Cl-GDY and HsGDY[72], Ghazzal and many scientists ${ }^{[73]}$ continued to study the chemical synthesis doping of GDY. Li et al. ${ }^{[72]}$ also gave full play to the fact that GDY could be prepared by chemical method, and adopted the 'bottom-up' hetero-atomic chemical doping synthesis strategy, through the chemical reduction and structural modification of the polymerization precursor, to prepare chlorine-doped GDY. The doped GDY materials prepared by chemical doping method have the characteristics of uniform distribution of heteroatoms and controllable content and doping position.

\subsubsection{Metal Element Doping}

Besides halogen heteroatom doping, there are also special interaction between metal atoms and GDY, metallic doped GDY can have obvious charge transfer effect. Doping metal atoms not only helps the preparation of GDY by certain coupling catalysis, but can effectively regulate the electronic and magnetic properties. Thus, improving or giving GDY new performance, provides the basis for its application. Metal atom (especially single-atom) doping has also been proposed to be a promising way for the modulation of GDY properties. GDY is considered as a good carrier for efficient and stable anchoring of metal atoms because of its excellent conjugate structure, large specific surface area and high adsorption energy for metal atoms ${ }^{[74-77]}$.

Based on the structural influence of the interaction

analyzed the N-type doping of metal atoms in the structure of metal atoms doped with GDY include $\mathrm{Au}, \mathrm{Cu}, \mathrm{Fe}, \mathrm{Pt}, \mathrm{Ni}, \mathrm{Mn}$, $\mathrm{Mo}, \mathrm{Ru}$, etc. ${ }^{[78-80]}$.

Transition metal doping can further improve the catalytic performance of graphite catalysts, in which Fe is the most effective metal element. Li et al. ${ }^{[81]}$ used Fe atoms to incorporate with GDY, endowing the magnetite/GDY heterojunctions with highly photocatalytic performances. Sun et al. ${ }^{[79]}$ revealed that GDY anchored with single $3 d$ transition-metal atom showed different structures and properties, which opened the way for the deeper cognation of GDY and its wider application.

\subsection{Click Chemistry Modification}

At present, there are few applications in the field of biology. In addition to the control problems and side reactions in the preparation, there are also the problems of poor dispersion and solubility of GDY nanoflakes in the aqueous solution system. When the size of the powders is small enough to a certain extent, the powders are easy to agglomerate, which makes the biological toxicity and biocompatibility of GDY worse. So, the solubility and well dispersion are the main bottlenecks that restrict the application of graphyne allotropes in the field of biology.

Therefore, in preparation, how to make the hydrophobic GDY stable in the body fluid environment dominated by aqueous solution is the first problem to be solved for the application of GDY in biology. Most of the methods, as mentioned above, to solve the problem of dispersion, solubility and compatibility of aqueous solution system are put forward to modify the GDY and expand its application scope.

One of the most commonly oxidation methods is to use a certain concentration of nitric acid or hydrochloric acid to oxidize GDY and participate in the application of biological design in the form of GDYO, as mentioned above. However, as the research on chemical mechanisms continues to deepen, there are more and more different ways to modify GDY. GDY consists of $s p$ and $s p^{2}$ hybridized carbon atoms with highly active $\pi$ bonds. The acetylene group on the edge of GDY can be used for further chemical derivation and modification. As reported in the literature ${ }^{[82]}$, the [3+2] Huisgen cycloaddition reaction catalyzed by copper(I) has been used for the preparation of side chain functional linear polymers. Therefore, it is an ideal method to modify GDY and improve its water solubility by quick click chemical synthesis using GDY endacetylene and azide compounds.

According to this, Li et al. ${ }^{[82]}$ functionalized GDY through 
Triazole was produced by Huisgen 1,3-dipole cycloaddition at the end of GDY and alkyl azide to obtain soluble GDY. Modified GDY had improved solubility and processability with remaining their intrinsic properties. The reaction could be carried out at a high yield under mild conditions without destroying the acetylene group. In addition, products with triazole bonds were extremely stable for hydrolysis, oxidation, or reduction.

The modified GDY not only significantly improves its solubility and processing performance, but also maintains its inherent properties, resulting in a high-quality film. Such soluble GDYs can provide promising applications for the development of GDY-based materials. This method is suitable for introducing a wide range of chemical functional structures.

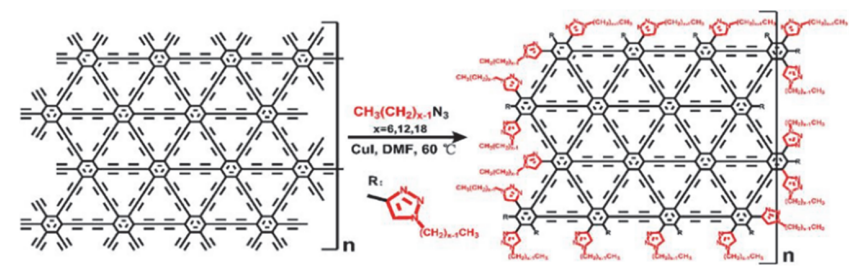

Fig.7 Schematic illustration of the chemical modification process of GDY[82]

Reprinted with permission from ref.[82]. Copyright 2019, Elsevier Ltd.

\section{Biomedical Applications of Graphdiyne}

GDY holds great promise for applications in batteries, solar cells, catalysis, and energy storage. As the increasing applications of GDY were reported, the biocompatibility of physicochemical, biological and mutagenic effects of GO and GDYO. The results showed that GO was only soluble in $\mathrm{H}_{2} \mathrm{O}$, but accumulated dynamically in $0.9 \% \mathrm{NaCl}$, phosphate buffered saline and cell culture medium, while GDYO could be well dissolved in various media. GO nanoparticles adhered and aggregated on the membrane of human hepatocyte cells, leading to cell stress and the production of reactive oxygen species, thus resulting in cell membrane wrinkle, methionine poisoning and apoptosis. In contrast, as shown in Fig.8, GDYO did not adhere to the cell membrane, confirming the biocompatibility of the GDY.

Table 1 summarizes the main applications of GDY in the biological field, which shows GDY has great application potential.

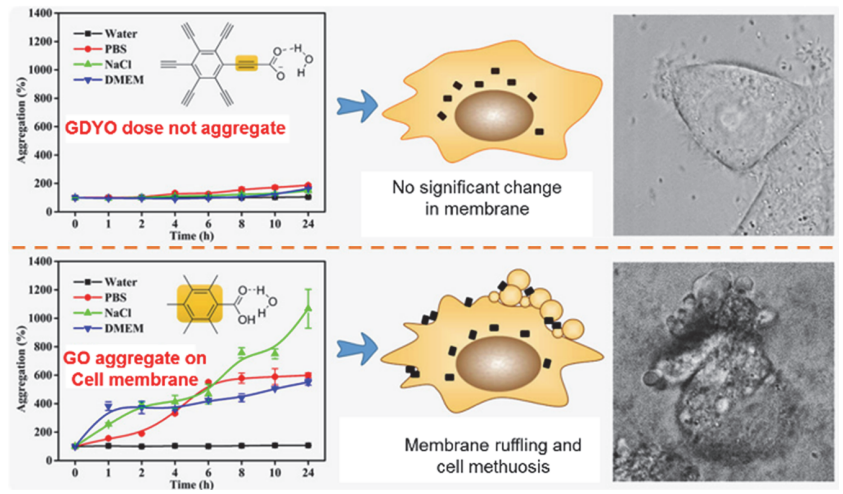

Fig.8 Comparisons between graphene oxide and graphdiyne oxide in physiochemistrybiology and cytotoxicity ${ }^{[59]}$

Reprinted with permission from ref.[59]. Copyright 2018, Amierican Chemical Society.

Table 1 Biological applications of GDY

\begin{tabular}{|c|c|c|c|}
\hline Method & Application & Unique advantage & Ref. \\
\hline $\begin{array}{l}\text { GDY-based sensing and } \\
\text { detection platform }\end{array}$ & $\begin{array}{l}\text { DNA } \\
\text { Micro RNA } \\
\text { Proteins } \\
\text { Amino acids } \\
\text { Glucose } \\
\mathrm{H}_{2} \mathrm{O}, \mathrm{Cu}^{2+}, \mathrm{H}_{2} \mathrm{O}_{2}\end{array}$ & $\begin{array}{l}\text { Nanopores } \\
\pi-\pi \text { stacking } \\
\text { Hydrophobic interaction } \\
\text { Attractive electronic properties, good Biocompatibility } \\
\text { High chemical and electrochemical stability }\end{array}$ & {$[12,54,57,83-100]$} \\
\hline Antibacterial & $\begin{array}{l}\text { Physical/mechanical } \\
\text { punctuations } \\
\text { Oxidative stress rupture } \\
\text { of cellular/membrane } \\
\text { Inhibition of bacterial } \\
\text { metabolism }\end{array}$ & $\begin{array}{l}\text { Wrapping: van der Waals/Hydrophobic interaction with bacterial membrane } \\
\text { Punctuation: Edge mechanical cutting damage } \\
\text { Strong oxidation ability to form higher valence band } \\
\text { Generation of ROS species } \\
\text { Highly active hybrid structure } \\
\text { Disturb multiple gene } \\
\text { All organic heterostructure }\end{array}$ & [101-107] \\
\hline GDY-based catalysts & $\begin{array}{l}\text { Nanozyme } \\
\text { GDY supported catalyst } \\
\text { GDY-based } \\
\text { semiconductor catalyst }\end{array}$ & $\begin{array}{l}\text { High specific surface area } \\
\text { Unique pore structure } \\
\text { Conjugated electronic structure } \\
\pi \text {-conjugate structure } \\
\text { Large specific surface area } \\
\text { Rich defect sites } \\
\text { Active alkyne bond } \\
\text { Rich electron } \\
\text { Active sp hybrid structure } \\
\text { Conjugated electronic structure } \\
\text { Hole-transfer material }\end{array}$ & [108-114] \\
\hline
\end{tabular}




\begin{tabular}{|c|c|c|c|}
\hline Method & Application & Unique advantage & Ref. \\
\hline \multirow[t]{14}{*}{ Tumor therapy } & Drug delivery carrier & $\pi$-conjugate structure & {$[58,115-126]$} \\
\hline & Nucleic acids delivery & Large specific surface area & \\
\hline & & Rich defect sites & \\
\hline & & Active alkyne bond & \\
\hline & & Active $s p$ hybrid structure & \\
\hline & Photothermal therapy & High photothermal conversion efficiency & \\
\hline & & Wide absorption spectrum & \\
\hline & & Excellent photoelectric response structure & \\
\hline & Photodynamic therapy & $\pi$-conjugate structure & \\
\hline & & Rich defect sites & \\
\hline & & Active alkyne bond & \\
\hline & & Active sphybrid structure & \\
\hline & Immunotherapy & High surface-to-volume ratios & \\
\hline & & GDYO-STAT3 interface: structure matching, hydrogen bonding, and salt bridges, & \\
\hline \multirow[t]{7}{*}{ Radiation protection } & Radiation protection in & High free radical scavenging ability & [127-129] \\
\hline & Radiotherapy & Unique optical properties & \\
\hline & & Good chemical stability and biosafety. & \\
\hline & UV protection & Ultrafast photoelectric characteristics & \\
\hline & & Unique optical nonlinear adsorption abilities & \\
\hline & & Large $\pi$-conjugated system & \\
\hline & & Excellent UV nonlinear characteristics(adsorb UV) & \\
\hline \multirow[t]{2}{*}{ Tissue engineering } & GDY-based artificial & Inherent network holes & {$[130,131]$} \\
\hline & synapse(GAS) & Rapid and selective transmission of diverse ions & \\
\hline
\end{tabular}

\subsection{Sensing and Detecting}

Alkynyl groups in GDY play important roles in enhancing sensing properties(Fig.9). Due to the planar structure and large surface area, GDY can easily capture biomolecules and maintain the maximum quenching efficiency of dyes. GDY also exhibits excellent sensing performances due to the light absorption covering from the UV-Vis region to the IR region. In addition, GDY is used as a support material for immobilizing enzymes to target biomolecules, thereby improving the selectivity of biosensors ${ }^{[33,84]}$.

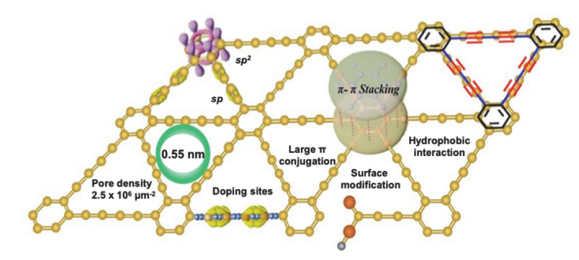

Fig.9 Characteristics of semiconductive GDY for biochemical sensing device ${ }^{[85]}$

Reprinted with permission from ref.[85]. Copyright 2020, Springer Nature.

\subsubsection{DNA Sensing Platform}

Benefiting from unique structure and properties, graphenebased devices have been applied for fast and cheap DNA sequencing technology. The density functional theory calculation indicated that the interaction between GDY and 6-carboxyfluorescein(FAM) was stronger than that of other 2D nanomaterials, such as graphene $e^{[85]}$, confirming that the presence of acetylene in GDY facilitated the single-stranded DNA(ssDNA) adsorption, which led to a further increase of fluorescence quenching ${ }^{[5,83]}$. In addition, GDY was used as a support material for immobilizing enzymes to target biomolecules, thereby improving the selectivity of electrochemical biosensors ${ }^{[84]}$.

Based on the mechanisms, such as fluorescence resonance energy transfer(FRET) or photoinduced electron transfer(PET), dye-labelled DNA was self-assembled onto the surface of GDY-based nano-quenchers by $\pi-\pi$ stacking and/or hydrophobic interaction. Wang et al. ${ }^{[54]}$ used oxidized GDY to establish a new platform for effective fluorescence sensing of DNA and thrombin(Fig.10). This study extends GDY to the field of fluorescence sensing for the first time, stimulating scientists' interest in 2D nanostructure fluorescence sensing.

Until 2019, Chang et al. ${ }^{[87]}$ reported a few layers of GDY NS $(0.9 \mathrm{~nm})$ as a fluorescence probe with ultra-high fluorescence quenching ability. It was found that the thinner the GDY, the stronger the fluorescence quenching effect. With the affinity difference for ssDNA and dsDNA, a method for $\mathrm{M}$. Tuberculosis(Mtb) and its drug resistance genes was developed. This sensing platform can be further applied for the Mtb detection fromclinical samples with a low background and a high signal-to-noise ratio.

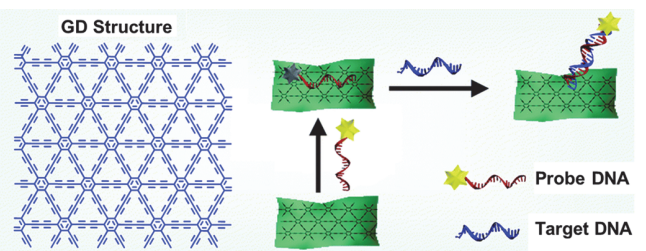

Fig.10 GDY-based fluorometric DNA assay ${ }^{[54]}$

Reprinted with permission from ref.[54]. Copyright 2016, Royal Society of Chemistry.

\subsubsection{Protein Sensor}

Enzymes and proteins play important roles in electron transfer, 
dioxygen binding, activation, and reduction ${ }^{[88]}$. Bisphenol $\mathrm{A}$ [2,2-bis(4-hydroxyphenyl) propane, BPA] is an important endocrine disruptor containing two hydroxyl groups, which can cause cardiovascular disease, carcinogenicity, neurotoxicity, and behavior problems. Tyrosinase, a coppercontaining protein, holds two distinct substrate binding sites for BPA and oxygen. Tyrosinase has high activity and specificity for BPA, which is more suitable for the construction of biosensors with high sensitivity and selectivity for the detection of BPA. Wu et al. ${ }^{[84]}$ fabricated GDY-based tyrosinase biosensor, which showed remarkable analytical performances for BPA detection with fast response, high sensitivity, good operation repeatability and low detection limit.

\subsubsection{MicroRNA Detector}

MicroRNA plays a key role in regulating human gene expression and widely exists in human tissues and body fluids. Therefore, ultrasensitive and feasible microRNA detection can achieve predictive diagnosis of a variety of human diseases, especially cancer ${ }^{[89,90]}$. Photoelectrochemical(PEC) methods featuring high sensitivity and feasibility might be a potential way to detecting MicroRNAs ${ }^{[12,92]}$. Compared with graphene, GDY has a naturally band gap of $1.12 \mathrm{eV}$, and possesses a high electron conductivity, manifesting that GDY has a promising application in PEC ${ }^{[3-95]}$.

Li et al. ${ }^{[132]}$ fabricated a photoactive material, which loaded GDY with AuNPs(AuNPs-GDY). AuNPs-GDY took advantage of the band-gap of GDY to produce hole-electron pairs, and the plasmon resonance effect of AuNPs to achieve a high PEC response. When the mass ratio of GDY to tetra-chloroauric acid was 1:2.5, AuNPs-GDY exhibited the best PEC performance. MicroRNA let-7a, a cancer marker, was chosen as a detection model. With a detection limit of $3.3 \times 10^{-19} \mathrm{~mol} / \mathrm{L}$ and a good linearity with microRNA concentration ranging from $1.0 \times 10^{-18}$ $\mathrm{mol} / \mathrm{L}$ to $1.0 \times 10^{-10} \mathrm{~mol} / \mathrm{L}$, this $\mathrm{PEC}$ biosensor provided a promising platform based on GDY to detect MicroRNA at ultralow levels for diagnoses.

\subsubsection{Other Biomolecule Detector}

Small biomolecules, such as carbohydrates and hydrogen peroxide are important biomarkers and mediators in many biological processes. Compared with graphene, GDY has larger pores composed of $\pi$-conjugated acetylenic bonds, which may have strong adsorption to biomolecules.

Amino acids detector: Chen et al. ${ }^{[1]}$ reported the interactions between single-layer GDY/Graphene sheet and Gly, Glu, His and Phe molecules. For each amino acid(AA) molecule, the adsorption energy on GDY is larger than that on graphene. Furthermore, the electrostatic polarization attractions with GDY are induced by strong electronegativity of $\mathrm{O}$ and $\mathrm{N}$ atoms in the AAs. The results proved that GDY was a promising $2 \mathrm{D}$ material for sensitively detecting amino acids and potentially used in bio-detectors.

Glucose detector: glucose plays an important role in the field of biology. It is the energy source and metabolic intermediate product of living cells, that is, the main energy supply of organisms. In the detection of diabetes, glucose is usually detected by enzyme, electrochemical detection and high performance liquid chromatography(HPLC) ${ }^{[133,134]}$.

Due to the high adsorption capacity and active sites on the GDY surface, Liu et al. ${ }^{[94]}$ prepared a GDY based glucose detection platform with dual enzyme activity by immobilizing ferrous ions and glucose oxidase on GDY tablets, providing a new perspective for the immobilization of ions and enzymes by 2D materials(Fig.11).

$\mathrm{H}_{2} \mathrm{O}_{2}$ detector: Hydrogen peroxide $\left(\mathrm{H}_{2} \mathrm{O}_{2}\right)$ is an important mediator in many biological processes. The conventional $\mathrm{H}_{2} \mathrm{O}_{2}$ detection methods include electrochemical method ${ }^{[98,99]}$, and coloring method ${ }^{[135]}$, etc.

Zhuang et al. ${ }^{[93]}$ reported an in situ synthesis of Prussian blue nanoparticles(PB) on GDYO. The PB/GDYO hybrid was used as an electrode with high electrochemical catalytic activity towards hydrogen peroxide. GDYO was able to anchor $\mathrm{PB}$ in the process of nanoparticle formation and stabilization. The PB/GDYO hybrid showed high electrochemical catalytic activity and stability for the detection of hydrogen peroxide.

Transition metal atoms can often be used as adsorption centers for small molecules. The $\pi$ bond on the surface of graphene is saturated and chemically inert, so the adsorption energy of metal atoms on graphene surface is less than $2 \mathrm{eV}$ and the migration barrier is less than $0.8 \mathrm{eV}^{[96,97]}$. Lower adsorption energy and migration barrier make metal atoms aggregate on GDY at room temperature. Therefore, GDY is easier to adsorb metal atoms than graphene, making it a potential molecular detection material.

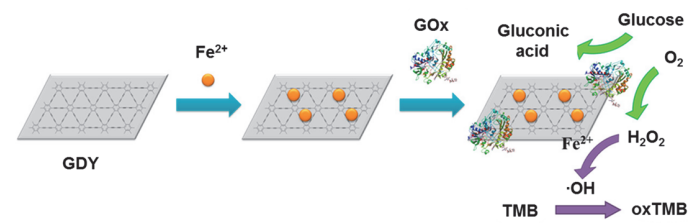

Fig.11 Scheme illustration of the one-step blood glucose detection based on GDY ${ }^{[94]}$

Reprinted with permission from ref.[94]. Copyright 2019, Amierican Chemical Society.

\subsubsection{Humidity and Respiration Sensor}

The unique acetylene nanostructure and strong hydrophobic skeleton make GDY a kind of new potential materials for $\mathrm{H}_{2} \mathrm{O}$ sensing. The necessary properties for humidity sensor include stable hydrophobic skeleton and extended hydrophilic 

with water molecules. This distinctive structure-based property enables the fabrication of a novel GDYO-based humidity sensor with an ultrafast response speed and good selectivity. Recently, Li et al. ${ }^{[136]}$ reported a new respiration monitor based on the GDY. The new GDY-based printed respiration sensor, based on the unique acetylene structure of GDY on the adsorption/desorption of water molecules, exhibited ultra-sensitive response and rapid recovery performance under real-time breathing conditions, and realized non-contact detection over a long distance of $40 \mathrm{~cm}$. This work opened the way for GDY as a new carbon-based ink for printing ultra-sensitive breathing sensors, which could be used to develop large-scale industrialized integrated intelligent breathing masks or skin monitors.

\subsection{6 $\mathrm{Cu}^{2+}$ Detector Through Click Chemistry}

As a ligand of various oxidoreductases and metalloproteins, $\mathrm{Cu}^{2+}$ is used in the metabolic process of human life. Developing a convenient and sensitive method to monitor $\mathrm{Cu}^{2+}$ in living cells or biological fluids is extremely vital for both scientific research and clinical diagnostics. Based on the excellent fluorescence quenching ability of GDY and the high specificity of click chemistry, a new sensing platform for sensitive detection of $\mathrm{Cu}^{2+}$ in living cells and body fluids was developed for the first time. In Fig.12, the $\mathrm{N}_{3}$ group of $\mathrm{N}_{3}$-dsDNA-6carboxyfluorescein(N-dsdna $\mathrm{N}_{3}$-dM) was specifically conjugated with the butyne group of GDY nanosheets(GDY NSs) by click chemistry in the presence of $\mathrm{Cu}^{2+}$ and $\mathrm{SA}$, so as to form a stable five membered triazole ring, expressed as triazole GDY. The proximity of FAM and GDY NSs promotes FRET from FAM to GDY NSs, resulting in fluorescence quenching of FAM. $\mathrm{Cu}^{2+}$ concentration was monitored by the change of fluorescence intensity(FL intensity). The ability of GDY to detect $\mathrm{Cu}^{2+}$ in living cells and human body fluids suggests its potential for screening $\mathrm{Cu}^{2+}$-related diseases.

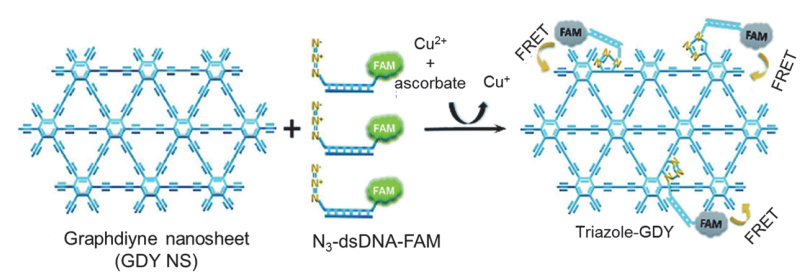

Fig.12 GDY NSs sensing platform for sensitive fluorescence detection of $\mathrm{Cu}^{2+}$ based on $\mathrm{Cu}^{+}$-catalyzed click chemistry ${ }^{[100]}$ Reprinted with permission from ref.[100]. Copyright 2021, Royal Society of Chemistry.

\subsection{GDY-based Imaging Probe}

\subsubsection{Fluorinated GDY}

Owing to the excellent extinction coefficient of GDY in the near-infrared region, GDY NSs have been explored as good imaging agents for cancer diagnose. The chemical modification of GDY using light elements is a possible route to regulate its unique structure and optoelectronic properties. Directly heating the mixture of xenon difluoride and GDY produces partially fluorinated GDY, whose covalent $\mathrm{C}-\mathrm{F}$ bond is hybridized with local $s p^{2}$ carbon because the alkyne bond is broken. It can be seen that the fluorescence of GDY is significantly enhanced due to the doping of fluorine. When the excitation wavelength varied from $260 \mathrm{~nm}$ to $480 \mathrm{~nm}$, the fluorinated GDY with different fluorine doping ratios showed photoluminescence from bright blue to green. In addition, GDY with fluorine content of $15.2 \%$ has strong photoluminescence properties and quantum efficiency of $3.7 \%$. The fluorescence enhancement is considered to be caused by defect states, which shows the potential properties of GDY as light-emitting devices, such as biosensors ${ }^{[37]}$.

\subsubsection{GDY Quantum Dots}

As a 0-dimensional nano material, GDY quantum dots (GDYQDs) have some unique advantages because of size effect, such as band gap generated by quantum confinement, good dispersion, richer active sites, biocompatibility, etc. Therefore, they are expected to break through the problem of GDY dispersion and dissolution and be applied to biological imaging and other fields. Experiments have proved that GDYQD has excellent light stability, can stimulate $\mathrm{pH}$ dependent fluorescence emission, has effective cell uptake and cell imaging ability, and will not induce detectable cytotoxic effects in vitro ${ }^{[138]}$.

Zhang et al. ${ }^{[139]}$ and Guo et al. ${ }^{[140]}$ combined GDY with functional chromophores with high chemical stability and quantum yield, such as pyrene, and synthesized GDY-Py QDs with strong fluorescence and good dispersion through the Sonogashira cross coupling reaction. It can be used for cell imaging and effectively overcomes the shortcomings of photobleaching with traditional fluorescent organic dyes. GDYQDs are expected to be used to construct high-quality fluorescent probes for biomedical localization and cell monitoring.

\subsection{Antibacterial Activity}

Because of the overuse of antibiotics in biomedicine and 

industry. The development of novel alternative antibacterial agents becomes extremely urgent.

2D nanomaterials can inhibit/kill microbial cells through a variety of mechanisms, including physical/mechanical puncture $^{[101-103]}$, oxidative stress ${ }^{[103]}$, cell/membrane component rupture $^{[104,105]}$ and inhibition of bacterial metabolism ${ }^{[106]}$. Liu et al. ${ }^{[111]}$ developed an efficient nanozyme constructed by $\mathrm{MoS}_{2} / \mathrm{rGO}$ with great capturing ability, as alternative antibiotics used in antibacterial field. Liu et al. ${ }^{[112]}$ have also constructed an all organic semiconductor heterostructure perylene-3,4,9,10-tetracarboxylic diimide(PDINH) based on $\mathrm{C}_{3} \mathrm{~N}_{4}$ for photocatalytic antibacterial. A series of experiments proved that all organic heterostructure had excellent inactivation effect on Gram-negative and positive bacteria, and its toxicity to normal tissue cells could be ignored. Therefore, as a kind of semiconductor, GDY itself possesses good biocompatibility, a chemically adjustable electronic structure, diverse structural flexibility and an appropriate band gap, which can construct a more broad-spectrum photocatalytic heterojunction with other organic semiconductors, so as to produce more reactive oxygen species for antibacterial. It also shows great application prospects in wound disinfection and effective promotion of infectious wound regeneration.

Zhu et al. ${ }^{[107]}$ studied the bacterial toxicity of GDY and GDYO, and further explored the antibacterial mechanism of GDY. The investigation of the antibacterial behaviors of GDY based nanomaterials may provide useful guidelines for the future design and application of this novel molecular allotrope of carbon ${ }^{[107]}$.

\subsubsection{Physical/Mechanical Punctuation}

Similar to the destructive effect of graphene on bacterial pathogen membrane ${ }^{[141,142]}$, GDY can also induce the collapse of bacterial cell membrane by capturing or wrapping bacterial cell membrane. Zhu et al. ${ }^{[107]}$ observed the formation of GDY and bacteria aggregation in bacterial culture. With the extension of culture time, the bacterial cell had no deformation meaning that wrapping bacterial membrane was a possible way to inhibit bacterial growth.

In addition to mechanical damage, graphene has a certain affinity for the phospholipid bilayer of the bacterial membrane, resulting in the collapse of the membrane. Similarly, GDY can also achieve antibacterial effect on the encapsulation and induction of bacterial membrane. The zeta potential of GDY in ultrapure water is $(2.1 \pm 0.3) \mathrm{mV}$, which is conducive to wrapping the negatively charged bacterial surface by electrostatic action. Moreover, the hydrophobic characteristics of GDY promote the hydrophobic interaction between GDY

Waals and hydrophobic interaction between GDY and bacterial membrane, which can damage the bacterial by wrapping or trapping bacterial membrane.

\subsubsection{Oxidative Stress Rupture of Cellular/Membrane Components}

Based on the unique semiconductor structure and alkyne bond system, the strong oxidation ability of GDY may contribute to the formation of higher valence band(VB) than GDYO. GDY can induce oxidative stress, interfere with bacterial metabolism and interrupt cell function. The antibacterial activity of GDY against Gram-positive bacteria was slightly higher than that of Gram-negative bacteria.

The antibacterial effect of GDY is not very strong through the chemical effect of oxidative stress ${ }^{[145,146]}$. Therefore, GDY composite antibacterial system is usually further constructed based on a unique large $\pi$-conjugated system and a highly active hybrid structure, such as modifying $\mathrm{TiO}_{2}$ nanofibers, using alkyne bond to bind photosensitive materials, such as zinc phthalocyanine and so on. Although $\mathrm{TiO}_{2}$ has photocatalytic activity for producing ROS, the recombination of electrons and holes limits its antibacterial ability. Wang et al. ${ }^{[147]}$ modified $\mathrm{TiO}_{2}$ nanofibers through GDY. The system had excellent biocompatibility and bone induction ability to induce cell adhesion and differentiation. The system not only improved the anti-drug-resistant-bacterial ability, but also helped promote the process of bone tissue regeneration.

\subsubsection{Inhibition of Bacterial Metabolism}

In order to understand the potential mechanism of antibacterial effect of GDY, as mentioned above, Zhu et al. ${ }^{[107]}$ explored the effect of GDY on the bacterial metabolism through differential gene expression analysis. The analysis of gene expression showed that the presence of GDY would disturb multiple genes responsible for bacterial metabolism. GDY could downregulate the metabolism-related genes and also compromise the bacterial membrane.

A combination of 'physical' and 'chemical' effects is accounted for the antimicrobial activity of GDY based nanomaterials. As shown in Fig.13, when bacteria directly contacted with GDY, GDY wrapped the bacterial membrane due to physical adsorption. At the same time, GDY NS has the function of blade similar to graphene nanosheet, whose sharp edge can be inserted into bacterial membrane, resulting in the leakage of substances in cells.

On the other hand, GDY may chemically induce the production of ROS, thereby interfering with specific microbial 


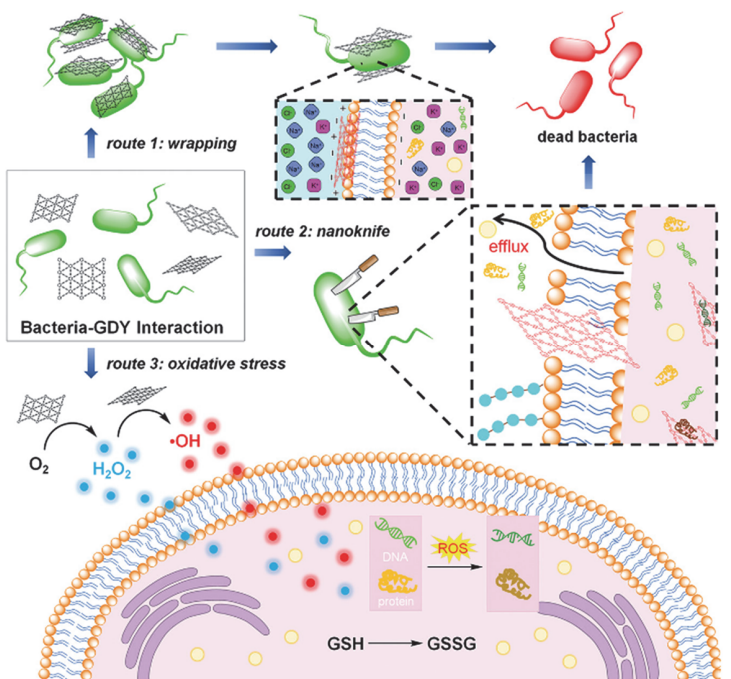

Fig.13 Possible antibacterial mechanisms of GDY-based nanomaterials ${ }^{[107]}$

Reprinted with permission from ref.[107]. Copyright 2020, John Wiley \& Sons, Inc.

processes. However, experiments showed that the 'physical' effect of GDY played a major role in the antibacterial process, and the 'chemical' effect of GDY had little effect on bacterial metabolism, which indicated that GDY induced oxidative stress was a secondary bactericidal factor.

\subsection{GDY-based Catalysts}

Unlike graphene, due to the hybridization of $s p$ and $s p^{2}$ in carbon atoms, GDY shows a unique semiconductor electron transport structure and a larger conjugate system. This phenomenon enhances the electron transport, which is not only conducive to the adsorption of reactants and intermediates, but also conducive to the fixation of single atoms on GDY, especially after doping modification. The formation of high active centers improves the catalytic performance of metals and their oxides. Therefore, GDY is expected to show better effects in the fields of catalysis, including photocatalysis, electrocatalysis and biocatalysis.

With the rapid development of nanoscience, some inorganic nanomaterials have been found to have enzyme-like catalytic activity. These nanomaterials can catalyze the substrate reaction of natural enzymes and have a catalytic mechanism similar to that of natural enzymes, so they are defined as nanozymes ${ }^{[108-110]}$.

However, due to their low catalytic activity, poor bacterial capturing capacity, and complicated material design, the feasibility of nanozymes is still far from satisfactory ${ }^{[111,112]}$. GDY with both $s p^{2}$ - and $s p$-hybridized carbon atoms is introduced to anchor ultrasmall nanoparticles with persistent enzyme-like activities. GDY is expected to become a nanozyme because of the high specific surface area, rich surface chemistry and easy functionalized structure. Ma et al..$^{[148]}$ developed the oxidized form of GDY, which could serve as a new kind of carbon nanozyme mimicking peroxidase. For the peroxidase-like activity of carbon-based nanomaterials, from the experimental and theoretical results, the ketone functional group unit was considered to be the catalytic center for $\mathrm{H}_{2} \mathrm{O}_{2}$ decomposition. Therefore, compared with GDY, GDYO with the oxygencontaining groups plays an important role in simulating the activity of peroxidase. Oxygen-containing groups are mainly used as active sites to absorb $\mathrm{H}_{2} \mathrm{O}_{2}$ and destroy $\mathrm{O}-\mathrm{H}$ bonds with $\mathrm{HO}_{2}{ }^{\bullet}$ and $\mathrm{H}^{+}$. In addition, the high specific surface area, unique pore structure and conjugated electronic structure of GDYO also provide rich transport channels and adsorption sites for the substrate ${ }^{[149-151]}$.

Zhou et al. ${ }^{[113]}$ developed a novel GDY, which can firmly anchor and disperse $\mathrm{CeO}_{2}$ nanoparticles to form GDY-CeO nanocomposites, exhibiting superior catalase-mimic activity in decomposing $\mathrm{H}_{2} \mathrm{O}_{2}$ to $\mathrm{O}_{2}$ and significantly alleviating tumor hypoxia, promoting radiation-induced DNA damage, and ultimately inhibiting tumor growth. Based on the high activity defect binding sites of GDY and the advantages of peroxidase simulation activity, Ali et al. ${ }^{[14]}$ developed a hemin/GDYhemin nanocomposite, which can efficiently catalyze the decomposition of $\mathrm{H}_{2} \mathrm{O}_{2}$ to produce highly toxic hydroxyl radical, damaging bacterial cell membrane, and finally leading to bacterial death in in vitro and in vivo experiments. The combination of hemin and GDY obviously overcome the agglomeration effect of hemin and significantly improved the effect. The results showed that GDY-hemin had excellent catalytic performance and good biocompatibility.

The 2D semiconductor properties of GDY provide good photogenerated electron-hole transmission ability ${ }^{[11,152]}$. With rich electron and good hole-transfer ability, GDY has great application potential in the field of photocatalysis. Using the adsorption capacity, which benefitted by its active defect sites, we can more effectively compared with traditional catalysts to prepare efficient semiconductor heterojunctions, which can be used not only for catalytic energy fields ${ }^{[153-155]}$, but also as a biological nanozyme with high catalytic activity for catalytic antibacterial and tumor treatment.

\subsection{Tumor Therapy}

The presence of high $\pi$-conjugation, numerous open cavities, and superior specific surface area make GDY an ideal delivery system for anticancer drugs. Based on these properties, GDY is used in a variety of tumor treatment strategies.

\subsubsection{Catalytic Therapy}

For solid tumors, the abnormally rapid value-added of cancer 
cells causes the core position of the tumor to become a low-oxygen environment. In the hypoxia environment, tumor cells are resistant to killing by ionizing radiation ${ }^{[15]}$. Therefore, increasing molecular oxygen content and lowering hypoxiainducing factor-1(HIF-1) levels can effectively inhibit tumor growth $^{[115]}$.

As shown in the Fig.14, Liu et al. ${ }^{[16]}$ prepared palladium nanoparticles PdNPs/GDY composite oxygen production platform by using the dispersed loading monatomic effect of GDY to reduce tumor hypoxia and inhibit tumor growth. In the presence of $\mathrm{H}_{2} \mathrm{O}_{2}$, the PdNPs/GDY decomposed $\mathrm{H}_{2} \mathrm{O}_{2}$ to produce molecular oxygen. After the oxygen content increased, HIF-1 was degraded, which efficiently delayed the growth of tumor in both human patient-derived squamous cell lung carcinoma xenograft model and 4T1 breast cancer xenografted mouse model. GDY, with abundant active sites and special adsorption capacity, plays a key role in the catalytic therapy of hypoxic tumor.

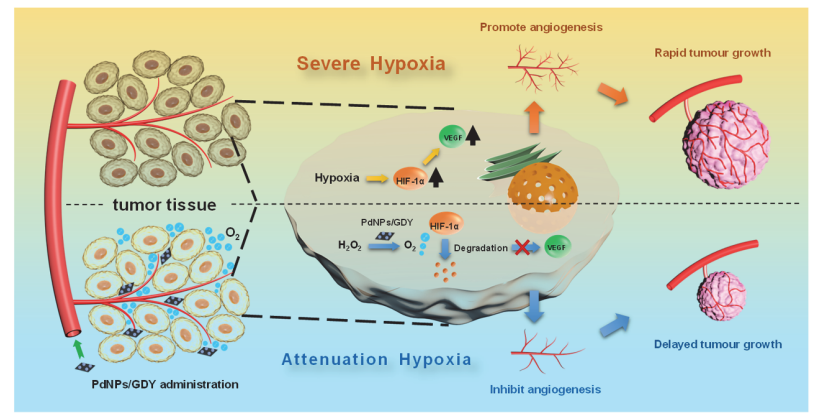

Fig.14 Schematic illustration of tumor catalytic therapy by attenuating hypoxia using PdNPs/GDY[116]

Reprinted with permission from ref.[116]. Copyright 2020, Elsevier Ltd.

\subsubsection{Photothermal Therapy}

Photothermal therapy(PTT) is an advanced type of localized tumor treatment ${ }^{[117]}$. GDY has high photothermal conversion efficiency and was recently used as a photothermal agent in PTT. Li et al. ${ }^{[118]}$ reported GDY NSs with PEGylation(GDY-PEG) as photothermal-acoustic wave nano-transducers for PTT in 2017 for the first time. To improve its biocompatibility, GDY was accompanied by PEGylation. Similar to other 2D materials, GDY-PEG had a broad optical absorption from the UV to the NIR regions. GDY-PEG also showed superior photostability with high photothermal conversion efficiency of $42 \%$, which was higher than that of other classic PTT agents ${ }^{[19,120]}$. Because of these excellent properties, GDY-PEG exerted an efficient antitumor effect at cellular level and xenograft model.

GDYO also had been used in PTT. Due to the high affinity of the carbon-carbon triple bonds in GDYO for iron, Min et al. ${ }^{[121]}$ invented a tumor-targeting iron sponge(TTIS) nanocomposite, with $\mathrm{Fe}_{3} \mathrm{O}_{4}$ nanoparticles deposited on the surface of GDYO. They also modified the nanosponge with the tumor targeting polymer, DSPE-PEG2000-CREKA. Thus, TTIS can accumulate in tumor cells and then elevate the temperature of tumor sites upon NIR irradiation, achieving an effective PTT.

\subsubsection{Photodynamic Therapy}

Photodynamic therapy is now well established and is used in the treatment of cancer and some superficial tumors, such as esophageal and bladder cancers ${ }^{[122-124]}$.

As mentioned above, Jiang et al. ${ }^{[58]}$ invented an iRGD peptide modified red blood cell membrane(i-RBM) coated GDYO nanosheet(GDYO@i-RBM), which could enhance oxygen generation in situ in tumor tissues, thereby significantly improving the effect of PDT treatment.

The researchers first successfully prepared GDYO nanosheets with a size of $(120 \pm 15) \mathrm{nm}$ and a thickness of $c a .1 .7$ $\mathrm{nm}$ through oxidation and exfoliation. Oxidation and exfoliation successfully controlled the energy band structure of GDY, significantly reduced the VBM, which gave GDYO a higher thermodynamic driving force for photooxidation and showed super high photocatalytic activity. After injection of GDYO@i-RBM nanosheets and irradiation by near-infrared light, the tumor blood vessels showed obvious expansion, and the hypoxia signal was significantly weakened. This research provides new inspiration for the design and synthesis of conjugated microporous nanocarriers in the future. What's more, it also further confirms that the modification of GDY can regulate the properties from the structure of the characterization and broaden the application fields.
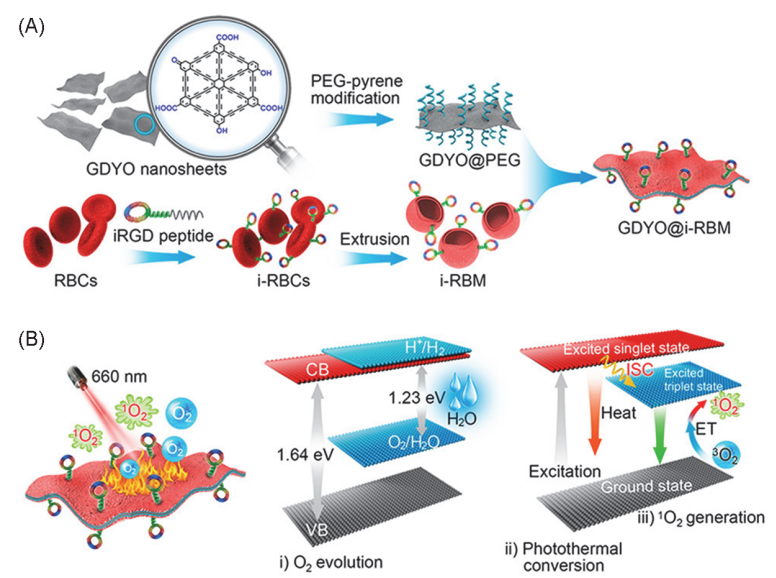

Fig.15 Schematic illustration of the synthetic process $(A)$ and working principles(B) of GDYO@i-RBM ${ }^{[58]}$

Reprinted with permission from ref.[58]. Copyright 2019, American Chemical Society.

\subsubsection{Immunotherapy}

Immunotherapy eliminates tumor cells by strengthening the body's own immune system, such as activating macrophages. 
Researches have shown that nanomaterials themselves can promote the release of inflammatory factors and induce M1 polarization of marophages ${ }^{[125,126,156]}$. Guo et al. ${ }^{[126]}$ studied the interaction between GDY/GDYO nanosheets and proteins in the macrophage cytoplasm and its subsequent regulation of macrophage behavior.

GDYO nanosheets were demonstrated to inhibit the immunosuppressive phenotype of tumor-associated macrophages(TAMs) by interaction with an intracellular protein-signal transducer and activator of transcription 3 (STAT3)(Fig.16), which could regulate the differentiation of macrophages. GDYO comprises of hybridized $s p$ and $s p^{2}$ carbon atom and the surface consists of well-arranged $\mathrm{C}=\mathrm{O}$ and $\mathrm{C}-\mathrm{OH}$ groups, making GDYO behave in an unpredictable manner at the nano-bio interface. The interaction at the GDYO-STAT3 interface, driven by structure matching, hydrogen bonding and salt bridges, regulated the immune response of macropages. For the first time, this study revealed an interaction mechanism between the nanoparticleprotein interfaces formed inside the cells, suggesting that GDYO nanosheets could structurally organize the intracellular protein corona to re-educate the suppressive capacities of TAMs, increase the infiltration of TILs, and sensitize the tumor environment toward immunotherapy.

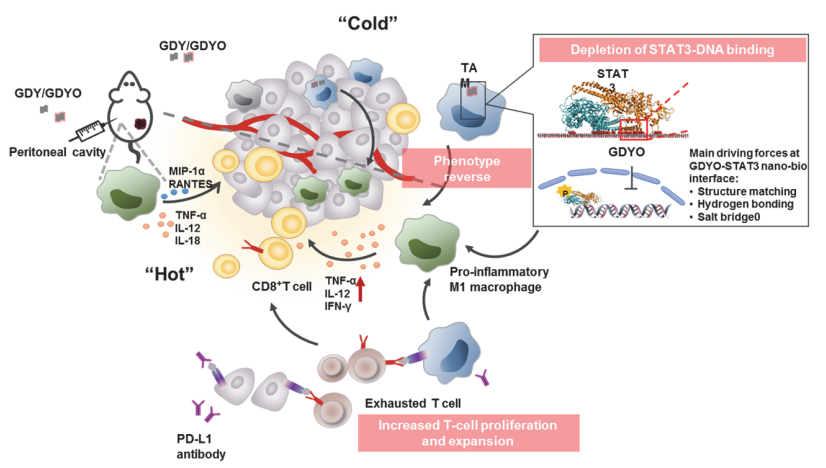

Fig.16 Schematic overview of the mechanism whereby GDYO nanosheets re-educate immunosuppressive macrophages through the intracellular corona and facilitate cancer immunotherapy ${ }^{[126]}$

Reprinted with permission from ref.[126]. Copyright 2021, American Chemical Society.

\subsection{Radiation Protection}

GDY possesses great fascination for radioprotection mainly due to its high free radical scavenging ability, unique optical properties, good chemical stability and good biocompatibility.

Radiotherapy has been widely used in cancer treatment because of its advantages of noninvasive and good effect. However, because the high-energy ionizing radiation inevitably damages normal cells, it is increasingly important to take radiation protection to avoid radiation damage.
Therefore, in recent years, many therapeutic systems for radiation protection have been developed by combining the design of nanomaterials with biosafety research. Many scholars have developed GDY-based radiation protective agents. For the first time, Xie et al. ${ }^{[127]}$ synthesized bovine serum albumin(BSA)-modified GDY nanoparticles(GDY-BSA NPs) to study the application of GDY for the radioprotection both in cell and in animal models. The results indicated that the GDYBSA NPs could effectively scavenge the DPPH, ABTS, $\mathrm{O}^{2-}$, and Ps. The research group further studied the effects of GDY-BSA NPs on gastrointestinal radiation protection ${ }^{[128]}$. The results further confirmed that GDY held the unique advantages of strong free radical scavenging ability, good chemical stability in gastric acid environment, long residence time in gastrointestinal tract and good biosafety through oral administration, which provided favorable preconditions for their application as gastrointestinal radiation protective agents.

In addition to the radiation of radiotherapy, ultraviolet is also a major killer of human cells. In recent years, some scholars ${ }^{[29]}$ have found that GDY had ultrafast photoelectric characteristics better than graphene, and possessed unique optical nonlinear adsorption abilities because of the unique $s p$ hybrid structure and large $\pi$-conjugated system. In particular, GDY has excellent UV nonlinear characteristics, which can just perfectly absorb UV.

\subsection{Tissue Engineering}

Tissue engineering refers to the use of artificial biological tissue to replace human tissue, exercising the structure and function. However, even in the field of more mature skin tissue engineering, the functional repair of the nervous system is still a difficult problem. Nervous system requires synapses to respond for receiving and transmitting electric signals. GDYbased artificial synapse(GAS), invented by Wei et al. ${ }^{[130]}$ in 2020, exhibited intrinsic short-term plasticity. GAS has been proposed to mimic the biological signal transmission behaviors. The relatively low diffusion barrier for ions in GDY benefited from its surface adsorption and interlayer insertion. The rapid diffusion and storage of ions profited from the existence of triangular pores in GDY structure and $s p$ hybrid carbon. At the same time, because of the good biocompatibility, GDY could bind to pre-synaptic neurons to form a hybrid system to achieve synaptic plasticity and complete the transmission of biological signal. Based on the previous work, Wei et al. ${ }^{[131]}$ connected gas with GDY based artificial muscle, completed the integration and output of artificial efferent nerve information, and opened up an interesting way for the later development and construction of GDY nerve. 


\subsection{Nucleic Acid Delivery}

Nucleic acids usually including DNA, mRNA, siRNA, miRNA and immunostimulatory nucleic acids, etc. are used to treat major diseases such as cancer ${ }^{[157]}$. Realizing the designed delivery of nucleic acids can refine the treatment to the gene level, but many levels of barriers in the biological environment must also be overcome in the process to deliver them to the cells. Common delivery challenges include large size, endosomal escape and release into cytoplasm, sensitivity to RNase and off-target immune stimulation(dsRNA). What all nucleic acids have in common are their strong negative charge and hydrophilicity, which make them cannot easily passthrough cell membranes. In order to be effectively and safely delivered to target cells, nucleic acids must usually be packaged in carriers, such as nanoparticles, which will allow them to be taken up by target cells and then released into appropriate cell compartments to function.

Nucleic acid vectors can be divided into viral and nonviral types, but due to the physiological toxicity and structural ambiguity of viruses, researchers are constantly developing non-viral vectors, such as liposomes and polymer vectors. Vaughan et al..157] summarized the development of nanomaterials for targeted nucleic acid delivery for the treatment of cancer. With the continuous development of nanotechnology, the research of nanomaterials as nucleic acid delivery vehicles is also constantly updated.

GDY, with $s p$ hybridized carbon atoms, a $\pi$-conjugate structure, a large specific surface area and an active alkyne bond, is a good type of nucleic acid delivery vector. As mentioned above, Zhou et al. ${ }^{[113]}$ used GDY to uniformly anchor $\mathrm{CeO}_{2}$ nanoparticles to form $\mathrm{GDY}-\mathrm{CeO}_{2}$, as an expedient MicroRNA vehicle(Fig.17), which could keep higher stability and efficiency to deliver miR181a to tumor, exhibiting low

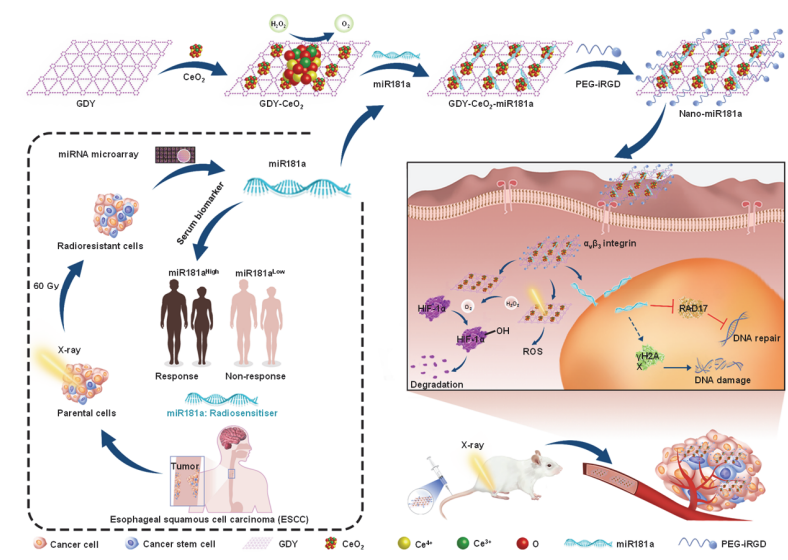

Fig.17 Multifunctional $\mathrm{GDY}^{-\mathrm{CeO}_{2}}$ nanozymes facilitating microRNA delivery and attenuating tumor hypoxia for highly efficient radiotherapy of esophageal cancer ${ }^{[113]}$

Reprinted with permission from ref.[113]. Copyright 2021, John Wiley \& Sons, Inc. could alleviate hypoxia by $\mathrm{H}_{2} \mathrm{O}_{2}$ oxidation in hypoxic tumor microenvironment, caused radiation-induced DNA damage, and ultimately inhibited tumor growth in vivo. This GDYbased nucleic acid delivery carbon nanomaterial platform further promotes the development of nanotechnology in the field of nucleic acid delivery.

\subsection{Vaccines and Vaccine Adjuvants}

At present, the New Coronary Pneumonia Epidemic is hitting the world, and the field of biomedicine is committed to the research and development of COVID-19 vaccines. As one of the most effective strategies to prevent and control infectious diseases and some non-communicable diseases, especially cancer, the design and development of vaccines are particularly critical. Appropriate addition of adjuvants and carriers to vaccine preparations can not only improve the immunogenicity of the antigen, but also induce long-term immunity.

Chen's group ${ }^{[158]}$ are also working on developing vaccine adjuvants, such as manganese adjuvants, using nanobiotechnology to improve vaccine efficacy. In recent years, researchers have also made research based on carbon nanomaterials ${ }^{[159,160]}$. Especially erewhile, researchers have tried to develop various functionalized GO vaccines and adjuvants based on the unique 2D properties of GO. Through adhesion and immune response experiments, Zhan et al. ${ }^{[161]}$ fully confirmed that GO nanosheets could be used as DC vaccine adjuvants and there was a size-dependent interaction between GO and DC cells, regulating the synapse formation of DC and DC-T cells, and promoting the production of powerful cytotoxic $\mathrm{T}$ cell immune response against SARS-CoV-2. This work provides new ideas for the development of effective COVID-19 vaccines using new two-dimensional carbon nanomaterials. As the same new type of 2D carbon nanomaterials, GDY and its oxidized derivatives are based on unique acetylene bonds and $s p$ hybrid carbons, which can be used as excellent nucleic acid delivery carriers, and they will also have the potential to be powerful for the development of new and efficient vaccines or vaccine adjuvants.

\section{Summary and Outlook}

Due to the hybrid structure and intrinsic hydrophobic properties of GDY, modification methods, such as oxidation and doping are still needed to develop to improve its hydrophilic, so as to increase its utilization efficiency.

In recent years, because of its unique structure and excellent performance, GDY has shown great potential in biological applications. First, due to its surface adsorption 
characteristics and conjugated structure, GDY can be used as a carrier in the fields of biocatalysis, drug delivery and so on. Then, the excellent photoelectric characteristics make GDY have unique performance in the design of biosensors and fluorescent probes. Third, after oxidative modification, the distribution of oxygen-containing functional groups on its surface was also found to be closely bound to the structure of tumor immune microenvironment related protein STAT3, which promoted the research on the mechanism and application of GDY in tumor immunotherapy. In addition, benefiting by the UV nonlinear absorption characteristics and free radical scavenging ability of GDY, it can be used in the field of UV and radiation protection. GDY possesses excellent semiconductor and photoelectric properties, which can also establish contact with neural synapses in tissue engineering. In conclusion, as a new 2D carbon nanomaterial, GDY has great application potential with its unique hybrid structure and advantages.

At present, the main factors limiting its application in the biological field are as follows:

(1) Material intrinsic limitations: as a new 2D carbon allotrope, GDY with high crystallinity has not been perfectly prepared. Researchers have also been limited to large-scale preparation of GDY. Besides, the experimental part is difficult to catch up with theoretical calculation. At present, the intrinsic problems of materials that limit their application, especially in the biological field, can be summarized as follows.

(a) The hydrophobicity of GDY leads to its poor solubility in the biological liquid environment. Therefore, most studies based on biological liquid environment are carried out by obtaining GDYO rich in hydroxyl and carboxyl groups after oxidation treatment.

(b) From the perspective of material preparation, the crystallinity of existing GDY is not high, and the quality needs to be improved. The defects of GDY may interfere with the cognition of practical application and mechanism exploration. Therefore, the research results so far cannot explain the specific action mechanism and effect of GDY, which also requires more efforts.

(2) Unclear Nano-Bio interaction mechanisms: nanomaterial-biological(Nano-Bio) interaction occurs at the interface between the surface of nanomaterials and surrounding biological liquids or biomolecules(such as proteins, DNA and lipids ${ }^{[162]}$. Because of its unique hybrid structure, porous structure, large specific surface area and excellent surface adhesion, GDY can specifically interact with biological molecules in the biological environment, but its nano-bio interaction mechanisms still remain unclear enough. In 2021, Guo et al. ${ }^{[126]}$ explored the intracellular protein corona(ICPC) of GDY. The researchers used the isotope ${ }^{13} \mathrm{C}$ to label GDYO, quantitatively analyzed the interaction ratio between GDYO and intracellular proteins. At the same time, the distribution and metabolism of GDYO in peritoneal macrophages and tumors after administration were analyzed, which provided a direct, accurate and quantitative analysis method for the assessment of the biological behavior of carbon nanomaterials in vivo.

Several typical studies based on theoretical simulation explored the functional mechanisms of the interaction between GDY and biological macromolecules in vivo. Through largescale all atom molecular dynamics simulation, Luan et al. ${ }^{[142]}$ revealed that GDY could indeed destroy the potential toxicity mechanism of protein-protein interaction(PPI) by cutting proteins, which provided a strong basis for reducing the toxic effect of GDY in biological cells. Zhang et al. ${ }^{[163]}$ used molecular dynamics simulation to explore the way to remove cholesterol from protein clusters with GDY as a promising carrier. This work first demonstrated the adhesion strength between a single cholesterol molecule and different types of raw graphite, which provided a basis for cholesterol interaction in protein cluster and dynamic cholesterol removal process. $s p$ hybrid carbon in GDY may be more active than $s p^{2}$ hybrid carbon in graphene, which makes GDY have significant affinity for cholesterol molecules. These findings opened up a promising way to make use of the biomedical application ability of GDY.

Although several progresses have been achieved, this burgeoning field is still evolving around many challenges. The relationships between the properties of GDY and the function are still not fully understood. The advanced technologies for dynamic and in situ detection of the nano-bio interaction process of GDY are still lacking.

The review of the physical properties, modification and the relative applications should be valuable to further studies. Synthesis decides the destiny. We believe that GDY and its derivatives have greater application potential in many fields, especially in the biological systems.

\section{Acknowledgements}

This work was supported by the Program for International S\&T Cooperation Projects of the Ministry of Science and Technology of China(Nos.2018YFE0117200, 2020YFA0710700), the Strategic Priority Research Program of Chinese Academy of Sciences(No.XDB36000000), the National Natural Science Foundation of China(No.11621505), the CAMS Innovation Fund for Medical Sciences (No.CIFMS2019-I2M-5-018), the Research and Development Project in Key Areas of Guangdong Province, China(No.2019B090917011) and the Strategic Priority Research Program of Chinese Academy of Sciences(No.XDB36000000).

\section{Conflicts of Interest}

The authors declare no conflicts of interest.

\section{References}

[1] Heimann R., Evsvukov S. E., Koga Y., Carbon, 1997, 35, 1654

[2] Enyashin A. N., Ivanovskii A. L., Physica Status Solidi(b), 2011, 248, 1879

[3] Kroto H. W., Heath J. R., O'Brien S. C., Curl R. F., Smalley R. E., Nature, 1985, 318, 162

[4] Lijima S., Nature, 1991, 354, 56 
[5] Novoselov K. S., Geim A. K., Morozov S. V., Jiang D., Zhang Y., Dubonos S. V., Grigorieva I. V., Firsov A. A., Science, 2004, 306, 666

[6] Bonaccorso F., Sun Z., Hasan T., Ferrari A. C., Nature Photonics, 2010, 4 611

[7] Zhang Y., Tang T., Girit C., Hao Z., Martin M. C., Zettl A., Crommie M. F., Shen Y. R., Wang F., Nature, 2009, 459, 820

[8] Zhou S., Gweon G. H., Fedorov A. V., First P. N., De Heer W. A., Lee D. H., Guinea F., Castro Neto A. H., Lanzara A., Nature Materials, 2007, 6, 770

[9] Baughman R. H., Eckhardt H., Kertesz M., The Journal of Chemical Physics, 1987, 87, 6687

[10] Haley M. M., Brand S. C., Pak J. J., Angewandte Chemie International Edition, 1997, 36, 836

[11] Long M., Tang L., Wang D., Li Y., Shuai Z., ACS Nano, 2011, 5, 2593

[12] Li G., Li Y., Liu H., Guo Y., Li Y., Zhu D., Chemical Communications, 2010, 46,3256

[13] Liu J., Chen C., Zhao Y., Advanced Materials, 2019, 31, e1804386

[14] Gao X., Liu H., Wang D., Zhang J., Chemical Society Reviews, 2019, 48,

[15] Cranford S. W., Brommer D. B., Buehler M. J., Nanoscale, 2012, 4, 7797

[16] Chen J., Xi J., Wang D., Shuai Z., The Journal of Chemical Physics, 2013 4,1443

[17] Kim B. G., Choi H. J., Physical Review B, 2012, 86, 115435

[18] Bai H., Zhu Y., Qiao W., Huang Y., RSC Advances, 2011, 1, 768

[19] Zhang Y. Y., Pei Q. X., Wang C. M., Applied Physics Letters, 2012, 101 081909

[20] Shao T., Wen B., Melnik R., Yao S., Kawazoe Y., Tian Y., The Journal of Chemical Physics, 2012, 137, 194901

[21] Koo J., Huang B., Lee H., Kim G., Nam J., Kwon Y., Lee H., The Journal of Physical Chemistry C, 2014, 118, 2463

[22] Zheng Y. P., Feng Q., Tang N. J., Du Y. W., New Carbon Materials, 2018 33, 516

[23] Chen Y., Liu H., Li Y., Chinese Science Bulletin, 2016, 61, 2901

[24] Luo G., Qian X., Liu H., Qin R., Zhou J., Li L., Gao Z., Wang E., Mei W. N., Lu J., Li Y., Nagase S., Physical Review B, 2011, 84, 075439

[25] Zheng Q., Luo G., Liu Q., Quhe R., Zheng J., Tang K., Gao Z., Nagase S. Lu J., Nanoscale, 2012, 4, 3990

[26] Luo G., Zheng Q., Mei W. N., Lu J., Nagase S., The Journal of Physical Chemistry C, 2013, 117, 13072

[27] Ge C., Chen J., Tang S., Du Y., Tang N., ACS Applied Materials \& Interaces, 2019, 11, 2707

[28] Sun T., Gao F. Y., Tang X. L., Yi H. H., Yu Q. J., Zhao S. Z., Xie X. Z., New Carbon Materials, 2021, 36, 304

[29] Coluci V. R., Braga S. F., Legoas S. B., Galvão D. S., Baughman R. H., MRS Online Proceedings Library, 2003, 739, 56

[30] Kong Y., Li J., Zeng S., Yin C., Tong L., Zhang J., Chem, 2020, 6, 1933

[31] Tahara K., Yoshimura T., Sonoda M., Tobe Y., Williams R. V., The Journal of Organic Chemistry, 2007, 72, 1437

[32] Gaab K. M., Thompson A. L., Xu J., Martínez T. J., Bardeen C. J., Journal of the American Chemical Society, 2003, 125, 9288

[33] Clair S., De Oteyza D. G., Chemical Reviews, 2019, 119, 4717

[34] Zhang Y. Q., Kepčija N., Kleinschrodt M., Diller K., Fischer S., Papageorgiou A. C., Allegretti F., Björk J., Klyatskaya S., Klappenberger F., Ruben M., Barth J. V., Nature Communications, 2012, 3, 1286

[35] Zuo Z., Shang H., Chen Y., Li J., Liu H., Li Y., Li Y., Chemical Communications, 2017, 53, 8074

[36] Qian X., Liu H., Huang C., Chen S., Zhang L., Li Y., Wang J., Li Y., Scientific Reports, 2015, 5, 7756

[37] Gao X., Zhu Y., Yi D., Zhou J., Zhang S., Yin C., Ding F., Zhang S., Yi X., Wang J., Tong L., Han Y., Liu Z., Zhang J., Science Advances, 2018, 4, eaat6378

[38] Gao X., Li J., Du R., Zhou J., Huang M. Y., Liu R., Li J., Xie Z., Wu L. Z., Liu Z., Zhang J., Advanced Materials, 2017, 29, 1605308

[39] Zhou J., Gao X., Liu R., Xie Z., Yang J., Zhang S., Zhang G., Liu H., Li Y., Zhang J., Liu Z., Journal of the American Chemical Society, 2015, 137 7596

[40] Yu H., Xue Y., Li Y., Advanced Materials, 2019, 31, 1803101

[41] Zhou J., Xie Z., Liu R., Gao X., Li J., Xiong Y., Tong L., Zhang J., Liu Z., ACS Applied Materials \& Interfaces, 2019, 11, 2632

[42] Wu J., Liang J., Zhang Y., Zhao X., Yuan C., Chemical Communications, 2021, 57, 5036

[43] Zhao F., Wang N., Zhang M., Sápi A., Yu J., Li X., Cui W., Yang Z., Huang C., Chemical Communications, 2018, 54, 6004

[44] Matsuoka R., Sakamoto R., Hoshiko K., Sasaki S., Masunaga H., Nagashio K., Nishihara H., Journal of the American Chemical Society, 2017, 139, 3145

[45] Yin C., Li J., Li T., Yu Y., Kong Y., Gao P., Peng H., Tong L., Zhang J., Advanced Functional Materials, 2020, 30, 2001396
[46] Yan H., Yu P., Han G., Zhang Q., Gu L., Yi Y., Liu H., Li Y., Mao L., Angewandte Chemie International Edition, 2019, 58, 746

[47] Mirnezhad M., Ansari R., Rouhi H., Seifi M., Faghihnasiri M., Solid State Communications, 2012, 152, 1885

48] Tan J., He X., Zhao M., Diamond and Related Materials, 2012, 29, 42

[49] Autreto P. A. S., De Sousa J. M., Galvao D. S., Carbon, 2014, 77, 829

[50] Li Y., Li Y., Acta Physico-Chimica Sinica, 2018, 34, 992

[51] Zhang P., Ma S., Sun L. Z., Applied Surface Science, 2016, 361, 206

[52] Mohajeri A., Shahsavar A., Computational Materials Science, 2016, 115, 51

[53] Mohajeri A., Shahsavar A., Journal of Materials Science, 2017, 52, 5366

[54] Wang C., Yu P., Guo S., Mao L., Liu H., Li Y., Chemical Communications, 2016, 52, 5629

[55] Li J., Chen Y., Guo J., Wang F., Liu H., Li Y., Advanced Functional Materials, 2020, 30, 2004115

[56] Huang C., Li Y., Wang N., Xue Y., Zuo Z., Liu H., Li Y., Chemical Reviews, 2018, 118, 7744

[57] Yan H., Guo S., Wu F., Yu P., Liu H., Li Y., Mao L., Angewandte Chemie International Edition, 2018, 57, 3922

[58] Jiang W., Zhang Z., Wang Q., Dou J., Zhao Y., Ma Y., Liu H., Xu H., Wang Y., Nano Letters, 2019, 19, 4060

[59] Zheng T., Gao Y., Deng X., Liu H., Liu J., Liu R., Shao J., Li Y., Jia L., ACS Applied Materials \& Interfaces, 2018, 10, 32946

[60] Koo J., Hwang H. J., Huang B., Lee H., Lee H., Park M., Kwon Y., Wei S. $\mathrm{H}$., Lee H., The Journal of Physical Chemistry C, 2013, 117, 11960

[61] He J., Wang N., Yang Z., Shen X., Wang K., Huang C., Yi Y., Tu Z., Li Y., Energy \& Environmental Science, 2018, 11, 2893

[62] Kim S., Lee J. Y., Journal of Colloid and Interface Science, 2017, 493, 123

[63] Kong X., Chen Q., Sun Z., RSC Advances, 2013, 3, 4074

[64] Lu R., Rao D., Meng Z., Zhang X., Xu G., Liu Y., Kan E., Xiao C., Deng K., Physical Chemistry Chemical Physics, 2013, 15, 16120

[65] Bu H., Zhao M., Zhang H., Wang X., Xi Y., Wang Z., Journal of Physical Chemistry A, 2012, 116, 3934

[66] Zhang S., Cai Y., He H., Zhang Y., Liu R., Cao H., Wang M., Liu J., Zhang G., Li Y., Liu H., Li B., Journal of Materials Chemistry A, 2016, 4, 4738

[67] Li J., Zhang Z., Kong Y., Yao B., Yin C., Tong L., Chen X., Lu T., Zhang J., Chem, 2021, 7, 1284

[68] Zou L., Zhu Y., Cen W., Jiang X., Chu W., Applied Surface Science, 2021, 557,149815

[69] Liu Q., Cheng L., Liu G., Membranes, 2020, 10, 286

[70] Kang B., Shi H., Wang F. F., Lee J. Y., Carbon, 2016, 105, 156

[71] Bhattacharya B., Singh N. B., Sarkar U., International Journal of Quantum Chemistry, 2015, 115, 820

[72] Wang N., He J., Tu Z., Yang Z., Zhao F., Li X., Huang C., Wang K., Jiu T., Yi Y., Li Y., Angewandte Chemie International Edition, 2017, 56, 10740

[73] Li J., Slassi A., Han X., Cornil D., HaThi M. H., Pino T., Debecker D. P., Colbeau Justin C., Arbiol J., Cornil J., Ghazzal M. N., Advanced Functional Materials, 2021, 31, 2170210

74] Lin Z. Z., Carbon, 2015, 86, 301

[75] Ma D. W., Li T., Wang Q., Yang G., He C., Ma B., Lu Z., Carbon, 2015, 95, 756

[76] Li J., Gao X., Jiang X., Li X. B., Liu Z., Zhang J., Tung C. H., Wu L. Z., ACS Catalysis, 2017, 7, 5209

[77] Mashhadzadeh A. H., Vahedi A. M., Ardjmand M., Ahangari M. G., Superlattices and Microstructures, 2016, 100, 1094

[78] Lin Z., Wei Q., Zhu X., Carbon, 2014, 66, 504

[79] He J., Zhou P., Jiao N., Ma S. Y., Zhang K. W., Wang R. Z., Sun L. Z., Scientific Reports, 2014, 4, 4014

[80] Pan C., Wang C., Fang Y., Zhu Y., Deng H., Guo Y., Environmental Science: Nano, 2021, 8, 1863

[81] Fang Y., Xue Y., Hui L., Yu H., Li Y., Angewandte Chemie International Edition, 2021, 60, 3170

[82] Chen Y., Li J., Wang F., Guo J., Jiu T., Liu H., Li Y., Nano Energy, 2019, 64, 103932

[83] Zhang Y., Xie Q., Xia Z., Gui G., Deng F., Journal of Electroanalytical Chemistry, 2020, 863, 114058

[84] Wu L., Gao J., Lu X., Huang C., Dhanjai, Chen J., Carbon, 2020, 156, 568

[85] Li J., Wan C., Wang C., Zhang H., Chen X., Chemical Research Chinese Universities, 2020, 36(4), 622

[86] Xiao J., Liu Z., Li C., Wang J., Huang H., Yi Q., Deng K., Li X., Analytical and Bioanalytical Chemistry, 2021, 413, 3847

[87] Chang F., Huang L., Guo C., Xie G., Li J., Diao Q., ACS Applied Materials \& Interfaces, 2019, 11, 35622

[88] Gaggelli E., Kozlowski H., Valensin D., Valensin G., Chemical Review, 2006, 106, 1995

[89] Li X., Li Y., Zhang J., Meng Y., Yu X., Wang X., Hun X., Sensors and Actuators B: Chemical, 2019, 297, 126808 
[90] Dave V. P., Ngo T. A., Pernestig A. K., Tilevik D., Kant K., Nguyen T., Wolff A., Bang D. D., Laboratory Investigation, 2019, 99, 452

[91] Chen X., Gao P., Guo L., Zhang S., Scientific Reports, 2015, 5, 16720

[92] Chang F., International Journal of Electrochemical Science, 2017, 10348

[93] Zhuang X., Mao L., Li Y., Electrochemistry Communications, 2017, 83, 96

[94] Liu J., Shen X., Baimanov D., Wang L., Xiao Y., Liu H., Li Y., Gao X., Zhao Y., Chen C., ACS Applied Materials \& Interfaces, 2019, 11, 2647

[95] Lin L., Liu Y., Zhao X., Li J., Analytical Chemistry, 2011, 83, 8396

[96] Ding J., Qiao Z., Feng W., Yao Y., Niu Q., Physical Review B, 2011, 84, 195444

[97] Li K., Li Y., Tang H., Jiao M., Wang Y., Wu Z., RSC Advances, 2015, 5, 16394

[98] Chen S., Yuan R., Chai Y., Hu F., Microchimica Acta, 2013, 180, 15

[99] Chen W., Cai S., Ren Q. Q., Wen W., Zhao Y. D., Analyst, 2012, 137, 49

[100] Ge C., Li J., Wang D., Lv K., Liu Q., Shen Y., Zhuang X., Luo W., Wu Z., Zhang Y., Shi L., Liu L., Bao S., Zhang H., RSC Advances, 2021, 11, 5320

[101] Bandara C. D., Singh S., Afara I. O., Wolff A., Tesfamichael T., Ostrikov K., Oloyede A., ACS Applied Materials \& Interfaces, 2017, 9, 6746

[102] Lu X., Feng X., Werber J. R., Chu C., Zucker I., Kim J. H., Osuji C. O. Elimelech M., Proceedings of the National Academy of Sciences of the united states of America, 2017, 114, E9793

[103] Linklater D. P., De Volder M., Baulin V. A., Werner M., JessI S., Golozar M., Maggini L., Rubanov S., Hanssen E., Juodkazis S., Ivanova E. P., ACS Nano, 2018, 12, 6657

[104] Zhu W., von demBussche A., Yi X., Qiu Y., Wang Z., Weston P., Hurt R. H., Kane A. B., Gao H., Proceedings of the National Academy of Sciences of the United States of America, 2016, 113, 12374

[105] Chen J., Zhou G., Chen L., Wang Y., Wang X. G., Zeng S.W., Journal of Physical Chemistry C, 2016, 120, 6231

[106] Xin Q., Liu Q., Geng L., Fang Q., Gong J., Advanced Healthcare Materials, 2017, 6

[107] Zhu Z., Bai Q., Li S., Li S., Liu M., Du F., Sui N., Yu W., Small, 2020, 16, e2001440

[108] Wu J., Wang X., Wang Q., Lou Z., Li S., Zhu Y., Qin L., Wei H., Chemical Society Reviews, 2019, 48, 1004

[109] Dugan L. L., Turetsky D. M., Du C., Lobner D., Wheeler M., Almli C. R., Shen C. K. F., Luh T. Y., Choi D. W., Lin T., Proceedings of the National Academy of Sciences, 1997, 94, 9434

[110] Gao L., Yan X., Science China Life Sciences, 2016, 59, 400

[111] Wang L., Gao F., Wang A., Chen X., Li H., Zhang X., Zheng H., Ji R., Li B. Yu X., Liu J., Gu Z., Chen F., Chen C., Advanced Materials, 2020, 32, 2005423

[112] Wang L., Zhang X., Yu X., Gao F., Shen Z., Zhang X., Ge S., Liu J., Gu Z., Chen C., Advanced Materials, 2019, 31, 1901965

[113] Zhou X., You M., Wang F., Wang Z., Gao X., Jing C., Liu J., Guo M., Li J. Luo A., Liu H., Liu Z., Chen C., Advanced Materials, 2021, 33, e2100556

[114] Ali A., Liu J., Zhou H., Liu T., Ovais M., Liu H., Rui Y., Chen C., Materials Chemistry Frontiers, 2021, 5, 6041

[115] Wilson W. R., Hay M. P., Nature Reviews Cancer, 2011, 11, 393

[116] Liu J., Wang L., Shen X., Gao X., Chen Y., Liu H., Liu Y., Yin D., Liu Y., Xu W., Cai R., You M., Guo M., Wang Y., Li J., Li Y., Chen C., Nano Today, 2020, 34, 100907

[117] Zhi D., Yang T., O'Hagan J., Zhang S., Donnelly R. F., Journal of Control Release, 2020, 325, 52

[118] Li S., Chen Y., Liu H., Wang Y., Liu L., Lv F., Li Y., Wang S., Chemistry of Materials, 2017, 29, 6087

[119] Hessel C. M., Pattani V. P., Rasch M., Panthani M. G., Koo B., Tunnell J. W., Korgel B. A., Nano Letters, 2011, 11, 2560

[120] Sun Z., Xie H., Tang S., Yu X. F., Guo Z., Shao J., Zhang H., Huang H., Wang H., Chu P. K., Angewandte Chemie International Edition, 2015, 54, 11526

[121] Min H., Qi Y., Zhang Y., Han X., Cheng K., Liu Y., Liu H., Hu J., Nie G., L Y., Advanced Materials, 2020, 32, 2000038

[122] Bechet D., Couleaud P., Frochot C., Viriot M. L., Guillemin F., BarberiHeyob M., Trends in Biotechnology, 2008, 26, 612

[123] Yi G., Hong S. H., Son J., Yoo J., Park C., Choi Y., Koo H., Quantative Imaging in Medicine and Surgery, 2018, 8, 433

[124] Fusco L., Gazzi A., Peng G., Shin Y., Vranic S., Bedognetti D., Vitale F., Yilmazer A., Feng X., Fadeel B., Casiraghi C., Delogu L. G., Theranostics, 2020, 10, 5435

[125] Zanganeh S., Hutter G., Spitler R., Lenkov O., Mahmoudi M., Shaw A., Pajarinen J. S., Nejadnik H., Goodman S., Moseley M., Coussens L. M., Daldrup Link H. E., Nature Nanotechnology, 2016, 11, 986

[126] Guo M., Zhao L., Liu J., Wang X., Yao H., Chang X., Liu Y., Liu J., You M., Ren J., Wang F., Wang L., Wang Y., Liu H., Li Y., Zhao Y., Cai R., Chen C., Nano Letters, 2021, 21, 6005

[127] Xie J., Wang N., Dong X., Wang C., Du Z., Mei L., Yong Y., Huang C., Li Y., Gu Z., Zhao Y., ACS Applied Materials \& Interfaces, 2019, 11, 2579

[128] Xie J., Wang C., Wang N., Zhu S., Mei L., Zhang X., Yong Y., Li L., Chen C., Huang C., Gu Z., Li Y., Zhao Y., Biomaterials, 2020, 244, 119940

[129] Yuan J., Liu G., Wang Z., Fu S., Ge X., Man Z., Han K., Zhang F., Xing F., Xu X., Journal of Materials Science, 2021, 56, 3653

[130] Wei H., Shi R., Sun L., Yu H., Gong J., Liu C., Xu Z., Ni Y., Xu J., Xu W., Nature Portfolio, 2020 DOI: 10.21203/rs.3.rs-53329/v1

[131] Wei H., Shi R., Sun L., Yu H., Gong J., Liu C., Xu Z., Ni Y., Xu J., Xu W., Nature Communications, 2021, 12, 1068

[132] Li Y., Li X., Meng Y., Hun X., Biosensors and Bioelectronics, 2019, 130 , 269

[133] Park S., Boo H., Chung T. D., Analytica Chimica Acta, 2006, 556, 46

[134] Alberti K. G., Zimmet P. Z., Diabet Medicine, 1998, 15, 539

[135] Wei H., Wang E., Analytical Chemistry, 2008, 80, 2250

[136] Li Y., Zhang M., Hu X., Yu L., Fan X., Huang C., Li Y., Nano Today, 2021, 39, 101214

[137] Xiao W., Kang H., Lin Y., Liang M., Li J., Huang F., Feng Q., Zheng Y., Huang Z., RSC Advances, 2019, 9, 18377

[138] Min H., Qi Y., Chen Y., Zhang Y., Han X., Xu Y., Liu Y., Hu J., Liu H., Li Y. Nie G., ACS Applied Materials \& Interfaces, 2019, 11, 32798

[139] Zhang D., Liu D., Li M., Yang Y., Wang Y., Yin H., Liu J., Jia B., Wu X., Analytica Chimica Acta, 2018, 1033, 180

[140] Guo J., Guo M., Wang F., Jin W., Chen C., Liu H., Li Y., Angewandte Chemie International Editor, 2020, 59, 16712

[141] MejíasCarpio I. E., Santos C. M., Wei X., Rodrigues D. F., Nanoscale, 2012, 4, 4746

[142] Luan B., Huynh T., Zhou R., The Journal of Physical Chemistry B, 2016 120, 2124

[143] Rowlett V. W., Mallampalli V., Karlstaedt A., Dowhan W., Taegtmeyer H., Margolin W., Vitrac H., Journal of Bacteriology, 2017, 199, https://doi.org/ 10.1128/JB.00849-16

[144] Gu Z., Yang Z., Luan B., Zhou X., Hong L., Zhou H., Luo J., Zhou R., The Journal of Physical Chemistry C, 2017, 121, 2444

[145] Liu Y., Zhao Y., Sun B., Chen C., Accounts of Chemical Research, 2013, 46,702

[146] Smith S. C., Rodrigues D. F., Carbon, 2015, 91, 122

[147] Wang R., Shi M., Xu F., Qiu Y., Zhang P., Shen K., Zhao Q., Yu J., Zhang $Y$, Nature Communications, 2020,11,4465

[148] Ma W., Xue Y., Guo S., Jiang Y., Wu F., Yu P., Mao L., Chemical Communications, 2020, 56, 5115

[149] Li Y., Zuo Z., Li Y., Handbook of Carbon-Based Nanomaterials, Elsevier Press, Amsterdam, 2021

[150] Jia Z., Li Y., Zuo Z., Liu H., Huang C., Li Y., Accounts of Chemical Research, 2017, 50, 2470

[151] Xue Y., Huang B., Yi Y., Guo Y., Zuo Z., Li Y., Jia Z., Liu H., Li Y., Nature Communications, 2018, 9, 1460

[152] Malko D., Neiss C., Viñes F., Görling A., Physical Review Letters, 2012 108,086804

[153] Lv J. X., Zhang Z. M., Wang J., Lu X. L., Zhang W., Lu T. B., ACS Applied Materials \& Interfaces, 2019, 11, 2655

[154] Yu H., Xue Y., Hui L., Zhang C., Li Y., Zuo Z., Zhao Y., Li Z., Li Y., Advanced Materials, 2018, 30, e1707082

[155] Covacci A., Telford J., Del Giudice G., Parsonnet J., Rappuoli R., Science, 1999, 284, 1328

[156] Peng G., Duan T., Guo M., Xue Y., Chen C., Li Y., Leifer K., Fadeel B., Nanoscale, 2021, 13, 13072

[157] Vaughan H. J., Green J. J., Tzeng S. Y., Advanced Materials, 2020, 32, 1901081

[158] Wang Y., Xie Y., Luo J., Guo M., Hu X., Chen X., Chen Z., Lu X., Mao L., Zhang K., Wei L., Ma Y., Wang R., Zhou J., He C., Zhang Y., Zhang Y., Chen S., Shen L., Chen Y., Quu N., Liu Y., Cui Y., Liao G., Liu Y., Chen C., Nano Today, 2021, 38, 101139

[159] Xu L., Xiang J., Liu Y., Xu J., Luo Y., Feng L., Liu Z., Peng R., Nanoscale, 2016, 8, 3785

[160] Cao W., He L., Cao W., Huang X., Jia K., Dai J., Acta Biomaterialia, 2020 112,14

[161] Zhou Q., Gu H., Sun S., Zhang Y., Hou Y., Li C., Zhao Y., Ma P., Lv L., Aji S., Sun S., Wang X., Zhan L., Advanced Materials, 2021, 2102528

[162] Wang Y., Cai R., Chen C., Accounts of Chemical Research, 2019, 52, 1507

[163] Zhang L., Wang X., RSC Advances, 2015, 5, 11776 